\title{
Perfect Reconstruction FIR Filter Banks: Some Properties and Factorizations
}

\author{
MARTIN VETTERLI, MEMBER, IEEE, AND DIDIER LE GALL, MEMBER, IEEE
}

\begin{abstract}
Perfect reconstruction FIR filter banks are analyzed both in $z$-transform and time domains, showing the alternatives between designs in the two domains. Various classes of perfect reconstruction schemes are indicated, and relations between previously known systems are given. Windowed modulated filter banks with low computational complexity and perfect reconstruction are shown. New factorizations of polyphase filter matrices, leading in particular to linear phase filters, are given. The computational complexity and the architecture of the new structures are indicated.
\end{abstract}

\section{INTRODUCTION}

A NALYSIS/SYNTHESIS systems like the one depicted in Fig. 1 and having the perfect reconstruction property can be regarded as generalized transforms where the "window" through which the input signal is seen is larger than the block size. Instead of processing separately adjacent blocks of the signal, such analysis/synthesis systems process overlapping blocks of the signal, thus reducing in part the problems inherent to block transform schemes. Such analysis/synthesis systems are used in subband coding methods [7] for speech and image compression, but regarded as generalized transforms, their use can be much broader.

The initial concern in these analysis/synthesis systems focused on aliasing cancellation since the multirate nature of such systems can lead to undesired aliased versions of the input signal in the output. The quadrature mirror filter solution in the two-channel case [8] solved this aliasing problem and soon became popular both in speech [7] and image [43] coding applications. The aliasing cancellation problem was then solved in the general case of an arbitrary number of channels [27], [31], [32], [45]-[47], [34]. The attention shifted to the perfect reconstruction issue. The initial solution for the two-channel case [29], [30], [16] was soon followed by solutions for an arbitrary number of channels [31], [32], [45]-[47], [34] and a time domain view of some of these results was given in [20], [25], [26]. It was then seen that the initial two-channel solution could be factored nicely into cascade forms [35],

Manuscript received May 3, 1988; revised October 26, 1988. The work of M. Vetterli was supported by the American National Science Foundation under Grants CDR-84-21402 and MIP-88-08277.

M. Vetterli is with the Department of Electrical Engineering and the Center for Telecommunications Research, Columbia University, New York, NY 10027.

D. LeGall is with Bell Communications Research, Morristown. NJ 07960 .

IEEE Log Number 8928132.
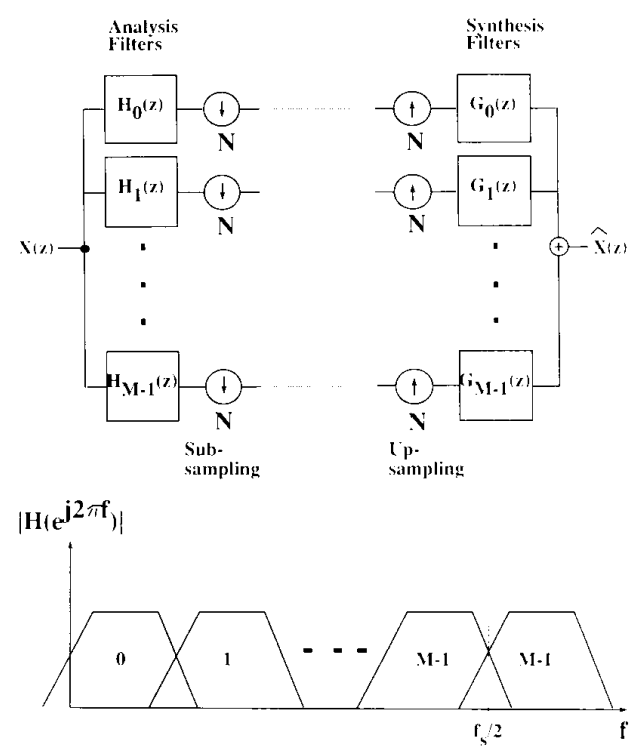

Fig. 1. Analysis/synthesis system with $M$ channels and subsampling by $N$. as well as typical frequency responses of the filters.

[39], a fact that holds more generally for a class of solutions [36], [38]. An excellent overview of the subject appeared in [37].

In parallel to the work on filter banks and associated perfect reconstruction analysis/synthesis systems, research on extending block transforms so as to include neighboring information led to the development of socalled lapped orthogonal transforms (LOT's) [4], [14] which use a window restricted to twice the transform size and guarantee perfect reconstruction.

The result of these various developments has been to state the perfect reconstruction filter bank problem as an analysis/synthesis problem of matrices of polynomials (in the $z$-transform domain) or of block-Toeplitz matrices (in the time domain) [20] with particular properties. This formulation establishes a close connection to the classical $N$-port synthesis problem in linear circuit theory [1], and this relation seems to be quite fruitful. Among the problems of continuing interest are the design of filter banks satisfying additional properties (like linear phase) as well as issues in computational complexity.

The outline of the paper is as follows. The basic equations for perfect reconstruction analysis/synthesis systems are given in Section II, pointing to the fundamental role 
played by polyphase filter matrices. Both time domain and $z$-transform domain analyses are used, and the relation between the two is shown as well. The two main representation forms for polyphase filter matrices, namely, the sum and the product form, are given in Section III, showing that the former has an easy time-domain interpretation, while the latter is better suited for cascade solutions. Section IV presents some solutions based on sum forms, in particular, modulated filter banks that allow windowing and achieve perfect reconstruction. Section V uses product forms to generate perfect reconstruction filter banks of arbitrary order meeting additional constraints like linear phase and/or modulation between filters. Of particular interest is the fact that while there is no linear phase paraunitary solution in the case $N=2$, such solutions exist for $N>2$. Finally, Section VI discusses the computational complexity as well as the possible architectures for the proposed filter banks.

\section{Analysis of Filter Banks and TRAnsform SySTEMS}

When analyzing filter banks or transform systems, one can use $z$-transform or time-domain methods. The former leads to matrices of polynomials (in the FIR case), while the latter gives rise to block-circulant or block-Toeplitz matrices. The two approaches are complementary, and given the problem at hand, one or the other might be better suited. We assume, following Fig. 1, that the analysis/ synthesis systems have $M$ channels (the number of channels is equivalent to the transform size) and that the channels are subsampled by $N$ (the step size at which the transform window advances over the signal). The case of most interest appears when critical sampling is used, i.e., the number of channels is equal to the subsampling factor $(M$ $=N$ ), and therefore the number of samples per unit of time is conserved in the system.

Since we are concerned mainly with perfect reconstruction analysis/synthesis systems where both the analysis and the synthesis filters are FIR with length $L_{a}$ and $L_{s}$, respectively, we will specifically look at the following problems.

- Is perfect FIR reconstruction possible?

- Is the complexity of the synthesis equal to the comIlexity of the analysis?

- Are the synthesis filters identical to the analysis filters (within possible time reversal)?

This will define classes of solutions as will be shown.

\section{A. z-Transform Analysis}

A filter with $z$-transform $H_{i}(z)$ followed by a subsampling by $N$ is best described by its decomposition into polyphase components $H_{i, k}\left(z^{N}\right)$ [3], [7], [37], [47].

$$
\begin{aligned}
H_{i}(z) & =\sum_{k=0}^{N-1} H_{i, k}\left(z^{N}\right) z^{-k} \\
H_{i, k}\left(z^{N}\right) & =\sum_{n=0}^{\infty} h_{i, k+n N} \cdot z^{-n N}
\end{aligned}
$$

where $h_{i, n}$ are the elements of the impulse response of the $i$ th filter. For example, a unit impulse at time $-k$ will generate an output in the subsampled domain equal to the $k$ th polyphase component, that is, $H_{i, k}(z)$. We define the following polyphase component matrix [37], [47] for the analysis filter bank:

$$
\begin{aligned}
& \boldsymbol{H}_{p}(z) \\
& =\left(\begin{array}{cccc}
H_{0.0}(z) & H_{0,1}(z) & \cdots & H_{0 . N-1}(z) \\
H_{1,0}(z) & H_{1.1}(z) & \cdots & H_{1, N-1}(z) \\
\vdots & \vdots & \cdot & \vdots \\
H_{M-1.0}(z) & H_{M-1,1}(z) & \cdots & H_{M-1 . N-1}(z)
\end{array}\right)
\end{aligned}
$$

and, with an inversion of the order of the polyphase components, the polyphase matrix for the synthesis filter bank:

$$
\begin{aligned}
& \boldsymbol{G}_{p}(z) \\
& \quad=\left(\begin{array}{cccc}
G_{0, N-1}(z) & G_{0, N-2}(z) & \cdots & G_{0,0}(z) \\
G_{1, N-1}(z) & G_{1, N-2}(z) & \cdots & G_{1,0}(z) \\
\vdots & \vdots & \cdot & \vdots \\
G_{M-1, N-1}(z) & G_{M-1, N-2}(z) & \cdots & G_{M-1,0}(z)
\end{array}\right) .
\end{aligned}
$$

It can be verified that a sufficient condition so that the analysis/synthesis system of Fig. 1 is a perfect reconstruction system is that [32], [34], [46], [47]

$$
\left[\boldsymbol{G}_{p}(z)\right]^{T} \cdot \boldsymbol{H}_{p}(z)=z^{-l} \cdot \boldsymbol{I} .
$$

Other solutions are obtained by pseudocirculant shifting of the identity matrix [40], and are therefore similar within a delay to the solution in (3). Note that the delay given by $z^{-l}$ on the right side of (3) is greater or equal to zero if all filters involved are causal. From the input to the output, there is an additional delay of $N-1$ samples due to the multirate nature of the system [47]. The design problem for perfect reconstruction systems is to find pairs of analysis and synthesis filter banks so that (3) is satisfied. Usually, the analysis bank is chosen first and then the synthesis bank is found so as to satisfy perfect reconstruction. Note that invertibility of $\boldsymbol{H}_{p}(z)$ is not sufficient since such an inverse might lead to unstable filters and because one might expect the synthesis filters to be FIR as well (assuming, as we do, that the analysis filters are FIR). Three classes of perfect FIR reconstruction systems can be defined.

I) Perfect FIR Reconstruction: The necessary and sufficient condition for FIR perfect reconstruction is that the determinant of $\boldsymbol{H}_{p}(z)$ be a monomial [46]. An equivalent statement is that the Smith form of $\boldsymbol{H}_{p}(z)$ [12] is a diagonal matrix of increasing delays. Then $G_{p}(z)$ can be obtained from the cofactor matrix of $\boldsymbol{H}_{p}(z)$ and will yield perfect reconstruction. 
2) Perfect FIR Reconstruction with Equal Length Analysis and Synthesis Filters: A sufficient condition for this class will be given in the next section.

3) Perfect FIR Reconstruction with Identical Analysis and Synthesis Filters: Note that the identity is within time reversal. A necessary and sufficient condition for this class is that $\boldsymbol{H}_{p}(z)$ satisfies

$$
\left[\boldsymbol{H}_{p}\left(z^{-1}\right)\right]^{T} \cdot \boldsymbol{H}_{p}(z)=\boldsymbol{I} .
$$

Obviously, in this case, $\boldsymbol{G}_{p}(z)$ can be chosen as

$$
\boldsymbol{G}_{p}(z)=z^{-m} \cdot \boldsymbol{H}_{p}\left(z^{-1}\right)
$$

where $m$ is chosen so that $\boldsymbol{G}_{p}(z)$ leads to causal synthesis filters, and therefore (3) is satisfied with $l=m$. Conversely, if $\boldsymbol{G}_{p}(z)$ satisfies (5), i.e., perfect reconstruction

$$
\boldsymbol{T}_{a}=\left(\begin{array}{ccccccc}
\ddots & \ddots & \ddots & \ddots & \ddots & \ddots & \ddots \\
\cdots & 0 & 0 & A_{0} & A_{1} & A_{2} & \cdots \\
\cdots & \cdots & 0 & 0 & A_{0} & A_{1} & A_{2} \\
\cdots & \cdots & \cdots & 0 & 0 & A_{0} & A_{1} \\
\ddots & \ddots & \ddots & \ddots & \ddots & \ddots & \ddots
\end{array}\right.
$$

is achieved with identical analysis and synthesis filter, then $\boldsymbol{H}_{p}(z)$ satisfies (4). A matrix $\boldsymbol{H}_{p}(z)$ that satisfies (4) is called a paraunitary or lossless (if it is stable) matrix [33], [34], [37], [41]. In the case of critical sampling ( $M$ $=N), \boldsymbol{H}_{p}(z)$ is square and the product in (4) is commutative.

$$
\boldsymbol{T}_{s}=\left(\begin{array}{ccccc}
\ddots & \ddots & \ddots & \ddots & \ddots \\
\cdots & 0 & 0 & \boldsymbol{B}_{K^{\prime}-1}^{T} & \cdots \\
\cdots & \cdots & 0 & 0 & \boldsymbol{B}_{K^{\prime}-1}^{T} \\
\cdots & \cdots & \cdots & 0 & 0 \\
\ddots & \ddots & \ddots & \ddots & \ddots
\end{array}\right.
$$

Obviously, class 1) contains 2) which in turn contains 3 ). While 3) is most desirable, it is also most constrained. Actually, we will see that certain design problems do not have a solution in 3). The other classes have more freedom, but also present more problems. In 1), the synthesis filters can be much longer than the analysis filters, and in both 1) and 2), the synthesis filters can have a somewhat exotic frequency response, even if the analysis filters are a perfectly well-behaved set of bandpass filters.

\section{B. Time-Domain Analysis}

The operation of a subsampled analysis filter bank can be described in the time domain with block-Toeplitz or block-circulant matrices (depending on how the boundaries are treated, but the operation is best seen as an infinite block-Toeplitz matrix acting on infinite signals). In the case of an $M$-channel filter bank with subsampling by $N$, the transformation of the inputs $x$ into the $M$ subsampled channels $y$ can be described according to the relation

$$
\boldsymbol{y}=\boldsymbol{T}_{a} \cdot \boldsymbol{x}
$$

where the input $\boldsymbol{x}$ is an infinite vector with time indexes

$$
\begin{aligned}
x= & {[\cdots, \cdots, x(-2), x(-1),} \\
& x(0), x(1), x(2), \cdots, \cdots],
\end{aligned}
$$

the output $y$ is an infinite vector consisting of $M$ multiplexed channels decimated by $N$

$$
\begin{aligned}
\boldsymbol{y}= & {\left[\cdots,\left[y_{0}(-N), y_{1}(-N), \cdots, y_{M-1}(-N)\right],\right.} \\
& {\left[y_{0}(0), y_{1}(0), \cdots, y_{M-1}(0)\right], } \\
& {\left.\left[y_{0}(N), y_{1}(N), \cdots, y_{M-1}(N)\right] \cdots\right], \quad(6 \mathrm{c}) }
\end{aligned}
$$

and the subscript $a$ in $T_{a}$ stands for analysis. The blocks in the matrix $T_{a}$ are of size $M$ by $N$, and the structure of the matrix $\boldsymbol{T}_{a}$ is of the form

$$
\left.\begin{array}{cccccc}
\ddots & \ddots & \ddots & \ddots & \ddots & \ddots \\
\boldsymbol{A}_{K-1} & 0 & 0 & \ldots & \ldots & \ldots \\
\cdots & \boldsymbol{A}_{K-1} & 0 & 0 & \ldots & \ldots \\
\boldsymbol{A}_{2} & \cdots & \boldsymbol{A}_{K-1} & 0 & 0 & \ldots \\
\ddots & \ddots & \ddots & \ddots & \ddots & \ddots
\end{array}\right)
$$

where each $\boldsymbol{A}_{i}$ is of size $M$ by $N$.

The reconstruction in the synthesis bank can be described by an inverse transformation

$$
\boldsymbol{x}=\boldsymbol{T}_{s} \cdot \boldsymbol{y} .
$$

Again, the matrix $\boldsymbol{T}_{s}$ has a block structure

$$
\left.\begin{array}{ccccccc}
\ddots & \ddots & \ddots & \ddots & \ddots & \ddots & \\
\boldsymbol{B}_{1}^{T} & \boldsymbol{B}_{0}^{T} & 0 & 0 & \ldots & \ldots & \ldots \\
\cdots & \boldsymbol{B}_{1}^{T} & \boldsymbol{B}_{0}^{T} & 0 & 0 & \ldots & \ldots \\
\boldsymbol{B}_{K^{\prime}-1}^{T} & \cdots & \boldsymbol{B}_{1}^{T} & \boldsymbol{B}_{0}^{T} & 0 & 0 & \cdots \\
\ddots & \ddots & \ddots & \ddots & \ddots & \ddots &
\end{array}\right)
$$

where $\boldsymbol{B}_{i}$ is of size $M$ by $N$ and the subscript $s$ stands for synthesis.

We assume now that $\boldsymbol{T}_{a}$ in (6) is a banded block-circulant matrix (circular extensions at boundaries) in order to avoid difficulties arising with infinite matrices. The weakest perfect reconstruction system occurs when the matrix $\boldsymbol{T}_{a}$ has full rank. The inverse matrix, in general, is not banded and this corresponds to perfect reconstruction with IIR filters (which might lead to stability problems). When the left inverse of $\boldsymbol{T}_{a}$ is also banded (the inverse is block-circulant [9]), we have perfect reconstruction with FIR filters. In the context of time-domain analysis, we find again three classes of perfect reconstruction systems with FIR filters. Class 1) corresponds to the case where the left inverse of $\boldsymbol{T}_{u}$ is banded and $K^{\prime}$ is arbitrary and can be much larger than $K$. Equal length analysis and synthesis filters $\left(K=K^{\prime}\right)$ corresponds to class 2$)$. Finally, if $\boldsymbol{T}_{a}$ is orthonormal (unitary), then

$$
\boldsymbol{T}_{a}^{T} \cdot \boldsymbol{T}_{a}=I
$$

and $\boldsymbol{B}_{i}=\boldsymbol{A}_{K-1-i}$, i.e., the synthesis filters are equivalent 
TABLE I

Properties of Perfect Reconstruction Systems in z-Transform and

Time Domains

\begin{tabular}{|l|l|c|}
\hline \hline Characteristic & z-domain & time domain \\
\hline a) aliasing cancellation & $\operatorname{Det} \mathbf{H}_{p}(z) \neq 0$ & Inverse of $\mathbf{T}_{a}$ exists \\
b) exact FIR reconstruction & $\operatorname{Det} \mathbf{H}_{p}(z)=$ delay & Inverse of $\mathbf{T}_{a}$ is banded \\
c) lapped transform & {$\left[\mathbf{H}_{p}(z)\right]^{-1}$ has same degree as $\mathbf{H}_{p}(z)$} & Inverse of $\mathbf{T}_{a}$ is banded and of same width \\
d) unitary solution & {$\left[\mathbf{H}_{p}\left(z^{-1}\right)\right]^{T} \cdot \mathbf{H}_{p}(z)=\mathbf{I}$} & {$\left[\mathbf{T}_{a}\right]^{T} \cdot \mathbf{T}_{a}=\mathbf{I}$} \\
e) lapped ortho. transform & same as above, plus add. constr. \\
\hline
\end{tabular}

to the analysis filters, within time reversal. Thus, we have a class 3 ) solution.

\section{Relationship Between z-Transform and Time-Domain Analysis}

In order to see the exact relationship between the timedomain and the $z$-transform analysis, it is convenient to express $\boldsymbol{H}_{p}(z)$ and $\boldsymbol{G}_{p}(z)$ as polynomials with matrix coefficients (rather than matrices with polynomial coefficients). We will call this the "sum form" of the polyphase filter matrices.

$$
\begin{aligned}
& \boldsymbol{H}_{p}(z)=\sum_{i=0}^{K-1} \boldsymbol{H}_{p i} \cdot z^{-i} \\
& \boldsymbol{G}_{p}(z)=\sum_{i=0}^{K^{\prime}-1} \boldsymbol{G}_{p i} \cdot z^{-i} .
\end{aligned}
$$

The values of the matrices $\boldsymbol{H}_{p i}$ and $\boldsymbol{G}_{p i}$ follow from (2) by inspection. Because of the time reversal inherent to the convolution, it is easy to verify that

$$
\boldsymbol{A}_{N-k-1}=\boldsymbol{H}_{p k} \cdot \boldsymbol{J}
$$

where $J$ is the antidiagonal matrix of size $N$ by $N$; note that this matrix $\boldsymbol{J}$ is essential and accounts for the difference between convolution (with time reversal) in (2) and matrix multiplication in (6). Similarly, because of the time reversal of the polyphase components of $G_{p}(z)$ in $(2 b)$, it turns out that

$$
\boldsymbol{B}_{k}=\boldsymbol{G}_{p k} \cdot \boldsymbol{J} .
$$

In summary, this section was used to set up the analysis framework for perfect reconstruction filter banks, and this both in $z$-transform and time-domain. Equation (10) establishes the relation between the time-domain block-circulant matrix of (6) and the polyphase matrix of (1). In Table I, the properties of exact reconstruction analysis/ synthesis systems are summarized both in the $z$-transform and time domain, and Fig. 2 shows the relation between the various classes.

\section{Sum Forms and Product Forms}

The sum form of (9) is an expression of the polyphase matrix $\boldsymbol{H}_{p}(z)$ that closely matches the time-domain representation of the filter bank. It will prove a particularly

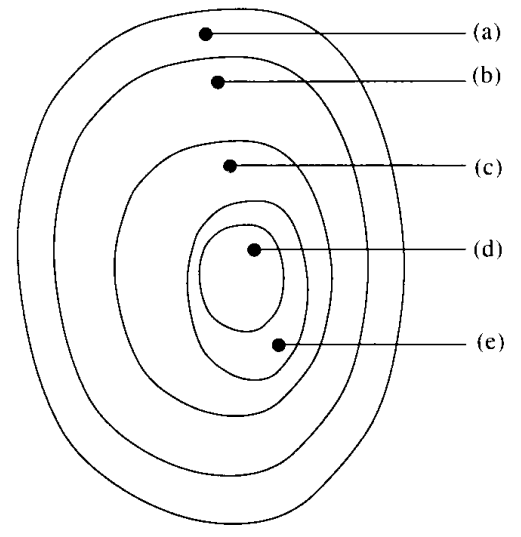

Fig. 2. Classes of perfect reconstruction filter banks. (a) Aliasing cancellation. (b) Perfect FIR reconstruction. (c) Lapped transforms $\left(L_{a}=L_{s}\right)$. (d) Paraunitary solutions. (e) Lapped orthogonal transforms

useful tool to relate the polyphase matrix representation to the time-domain properties.

As a simple example, take the case of the block transform of size $N$ by $N$ that advances by $N$ samples at a time over the signal. The matrix $\boldsymbol{T}_{a}$ in (6) is therefore block diagonal with block $\boldsymbol{A}_{0}$ of size $N$ by $N$. Assume that $\boldsymbol{A}_{0}$ is a unitary transform; then perfect reconstruction is achieved with $\boldsymbol{A}_{0}=\boldsymbol{B}_{0}$ and we have $\boldsymbol{T}_{a}^{-1}=\boldsymbol{T}_{a}^{T}$. In terms of $z$-transform analysis, we can write [from (10)]

$$
\begin{aligned}
& \boldsymbol{H}_{p}(z)=\boldsymbol{A}_{0} \cdot \boldsymbol{J} \\
& \boldsymbol{G}_{p}(z)=\boldsymbol{B}_{0} \cdot \boldsymbol{J}=\boldsymbol{A}_{0} \cdot \boldsymbol{J} .
\end{aligned}
$$

Therefore, replacing (11) in (3), we get

$$
\boldsymbol{J} \cdot \boldsymbol{A}_{0}^{T} \cdot \boldsymbol{A}_{0} \cdot \boldsymbol{J}=\boldsymbol{I}
$$

since $\boldsymbol{J}_{N}^{2}=\boldsymbol{I}$ and perfect reconstruction is verified. Note that this simple example shows also that block transform methods are a particular case of analysis/synthesis systems with filter length, number of channels, and subsampling factors all equal to $N$ (see Fig. 3). In this block transform case, the sum form is a matrix polynomial of degree zero.

A more general property based on sum forms is introduced by forcing the various matrices $\boldsymbol{A}_{i}$ or their associated $\boldsymbol{H}_{p i}$ to satisfy mutual orthogonality properties. Equal 


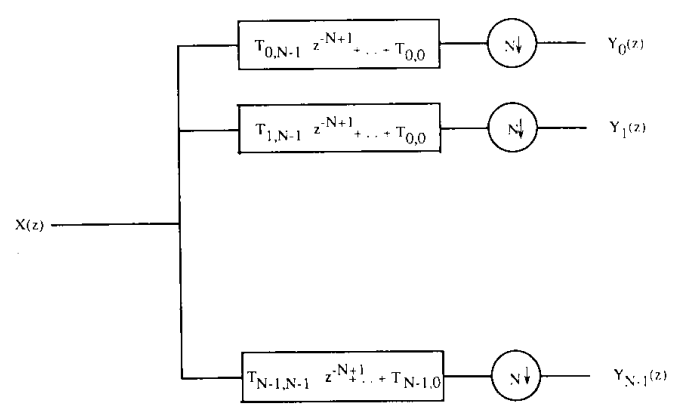

Fig. 3. Size- $N$ block transform interpretation as a filter bank with $N$ filters of length $N$ subsampled by $N$.

length analysis and synthesis filters $\left(K=K^{\prime}\right)$ can be achieved by the following sufficient condition [48].

Condition $\mathrm{Cl}$ : Orthogonality of overlapping blocks, that is,

$$
\sum_{i=0}^{K-1} \boldsymbol{A}_{i+k}^{T} \boldsymbol{A}_{i}=0, \quad k=1, \cdots, K-1
$$

is sufficient for perfect FIR reconstruction with equal length filters given that there are $N$ linearly independent analysis filters. This condition was called "orthogonality of the tails of the transform" in the case where the filters were restricted to $L=2 N$ [4], [14].

Proof: The product $\boldsymbol{T}_{a}^{T} \cdot \boldsymbol{T}_{a}$ is block diagonal because of (13) and the diagonal element is a matrix of size $N$ by $N$ equal to

$$
\boldsymbol{T}_{d}=\sum_{i=0}^{K-1} \boldsymbol{A}_{i}^{T} \cdot \boldsymbol{A}_{i}
$$

Since there are $N$ independent filters, $\boldsymbol{T}_{d}$ has rank $N$ and can thus be inverted. Note also that $\boldsymbol{T}_{d}$ is symmetric and positive definite. Then, the synthesis matrix $\boldsymbol{T}_{s}$ is of the form

$$
\begin{aligned}
\boldsymbol{T}_{s}= & \left(\begin{array}{ccccccc}
\ddots & \ddots & \ddots & \ddots & \ddots & \ddots & \ddots \\
\cdots & 0 & T_{d}^{-1} & 0 & \cdots & \cdots & \cdots \\
\cdots & \cdots & 0 & T_{d}^{-1} & 0 & \cdots & \cdots \\
\cdots & \cdots & \cdots & 0 & T_{d}^{-1} & 0 & \cdots \\
\ddots & \ddots & \ddots & \ddots & \ddots & \ddots & \ddots
\end{array}\right) \\
& \cdot T_{a}^{T}
\end{aligned}
$$

which can be expanded as

$$
\boldsymbol{T}_{s}=\left(\begin{array}{cccc}
\cdots & 0 & T_{d}^{-1} \cdot A_{K-1}^{T} & \cdots \\
\cdots & \cdots & 0 & T_{d}^{-1} \cdot A_{K-1}^{T} \\
\cdots & \cdots & \cdots & 0
\end{array}\right.
$$

and we verify that we have a class 2 ) solution and $K=$ $K^{\prime}$.

Q.E.D.

Note that the condition of orthogonality of the tails is not necessary since one can have different filters at the analysis and at the synthesis. This can lead to other solutions of interest as will be shown in Section V.
When the $N$ by $N$ matrix $T_{d}$ equals identity, we have the property

$$
T_{a}^{T} \cdot T_{a}=I
$$

and the synthesis filters are equivalent to the analysis filters. This means that the filters of the bank are mutually orthogonal and of unity norm. In the critically sampled case $M=N, T_{a}$ is square and therefore a unitary matrix.

Now, using the sum form, we can draw some parallels between the time domain and the $z$ domain. First, what is the meaning of condition $\mathrm{C} 1$ (orthogonality of the tails) in the $z$-transform domain? Let us consider the product

$$
\begin{aligned}
& {\left[\boldsymbol{H}_{p}\left(z^{-1}\right)\right]^{T} \cdot \boldsymbol{H}_{p}(z)} \\
& \quad=\sum_{j=-K+1}^{K-1} z^{j}\left(\sum_{i=j-K+1}^{j+K-1} \boldsymbol{H}_{p i}^{T} \cdot \boldsymbol{H}_{p(i-j)}\right) \\
& \quad=\sum_{i=0}^{K-1} \boldsymbol{H}_{p i}^{T} \cdot \boldsymbol{H}_{p i}=\boldsymbol{T}_{d}^{\prime} .
\end{aligned}
$$

This is because of (13), the orthogonality of the tails. Note that $\boldsymbol{T}_{d}^{\prime}=\boldsymbol{J} \cdot \boldsymbol{T}_{d} \cdot \boldsymbol{J}$ following (14) and (10a). Therefore, choosing $G_{p}(z)$ as

$$
\boldsymbol{G}_{p}(z)=z^{-K+1} \cdot \boldsymbol{H}_{p}\left(z^{-1}\right) \cdot\left[\boldsymbol{T}_{d}^{\prime}\right]^{-1}
$$

will yield perfect reconstruction and defines thus a class 2) solution in the $z$-transform domain.

Note that the $k$-indexed sequence $\boldsymbol{J} \cdot \Sigma_{i=0}^{K-1} \boldsymbol{A}_{i+k}^{T} \boldsymbol{A}_{i} \cdot \boldsymbol{J}$ is the (matrix) autocorrelation sequence [42] of the sequence $\boldsymbol{H}_{p}(k)$, i.e., $\Sigma_{i=0}^{K-1} \boldsymbol{H}_{p(i+k)}^{T} \boldsymbol{H}_{p i}$, leading to the interpretation that the orthogonality of the tail amounts to a (matrix) autocorrelation with only the central term different from zero.

Assume now that $\boldsymbol{T}_{d}^{\prime}=\boldsymbol{T}_{d}=\boldsymbol{I}$; then (17) means that $\boldsymbol{H}_{p}(z)$ satisfies (4). From (16) and (17), we have the equivalence

$$
\boldsymbol{T}_{a}^{T} \cdot \boldsymbol{T}_{a}=\boldsymbol{I} \Leftrightarrow\left[\boldsymbol{H}_{p}\left(z^{-1}\right)\right]^{T} \cdot \boldsymbol{H}_{p}(z)=\boldsymbol{I} .
$$

Thus, paraunitariness of $\boldsymbol{H}_{p}(z)$ is equivalent to $\boldsymbol{T}_{a}$ having orthonormal columns. We can also observe [42] that when $\boldsymbol{T}_{d}$ has rank $N$, then it is symmetric and positive definite and thus can be factorized into $\boldsymbol{T}_{d}=\boldsymbol{C}^{T} \cdot \boldsymbol{C}$. Normalization of the system by $C$ will then yield a paraunitary system.

The concept of orthogonal overlaps derived from the sum forms has been shown to be useful in order to derive

$$
\left.\begin{array}{ccccc}
\hline T_{d}^{-1} \cdot A_{0}^{T} & 0 & \cdots & \cdots & \cdots \\
\ldots & T_{d}^{-1} \cdot A_{0}^{T} & 0 & \cdots & \ldots \\
T_{d}^{-1} \cdot A_{K-1}^{T} & \ldots & T_{d}^{-1} \cdot A_{0}^{T} & 0 & \cdots
\end{array}\right)
$$

solutions that satisfy certain length constraints on the synthesis filters. It also leads to the proof of the equivalence between unitary time-domain matrices and paraunitary $z$-domain matrices.

The time domain or sum form expression for exact reconstruction with synthesis filters of the same length gives 
a good intuition of the phenomena involved, but when the degree of the sum form (9a) becomes large, the problem becomes intractable for large $K[(13)$ is nonlinear] and another approach will be necessary. For this reason, we will introduce product forms. We shall call the product form of the polyphase filter matrix the expansion [36]

$$
\boldsymbol{H}_{p}(z)=\boldsymbol{H}_{p 0} \cdot \prod_{i=1}^{K-1} D_{i}(z) \boldsymbol{H}_{p i}
$$

where the matrices $\boldsymbol{H}_{p i}$ have full rank and the matrices $D_{i}(z)$ are diagonal matrices of delays. The synthesis polyphase filter matrix can be expanded as

$$
\boldsymbol{G}_{p}(z)=\boldsymbol{G}_{p 0} \cdot \prod_{i=1}^{K-1} \boldsymbol{D}_{i}^{\prime}(z) \boldsymbol{G}_{p i} .
$$

Unlike the sum forms, the product forms do not always exist since (20)-(21) imply reconstruction by filters of the same length. On the other hand, if $\boldsymbol{H}_{p}(z)$ is paraunitary, the product form always exists [1], and conversely, if all the $H_{p i}$ are unitary matrices (rotations), then $\boldsymbol{H}_{p}(z)$ is paraunitary. Product forms allow for a cascade generation of filter banks satisfying a given property, for example, paraunitariness [36], [39], but also symmetries in the filter bank as will be shown in Section V. Nonparaunitary product forms have also been used in [50] to generate interesting design examples of perfect reconstruction filter banks.

\section{Sum Form Solutions in the Restricted Length CASE $L_{a}=2 N$}

When the analysis filters are restricted to $L_{a}=2 N$ (that is, the analysis window is twice the block size), the filter design problem is simplified and the results are still useful in applications like image processing where short filters are often used. Furthermore, this case is simple enough and should therefore illustrate some of the results from the previous section. Only the critically sampled case ( $M$ $=N$ ) will be considered, and same length analysis and synthesis filters are desired $\left(L_{a}=L_{b}=2 N\right)$.

\section{A. Analysis of the Case $L_{a}=2 N$}

From (6) and (7), we see that $\boldsymbol{T}_{s} \cdot \boldsymbol{T}_{a}=\boldsymbol{I}$ can be met by satisfying

$$
\begin{aligned}
& \boldsymbol{B}_{0}^{T} \cdot \boldsymbol{A}_{0}+\boldsymbol{B}_{1}^{T} \cdot \boldsymbol{A}_{1}=\boldsymbol{I} \\
& \boldsymbol{B}_{0}^{T} \cdot \boldsymbol{A}_{1}=\boldsymbol{B}_{1}^{T} \cdot \boldsymbol{A}_{0}=\mathbf{0} .
\end{aligned}
$$

Orthogonality of the overlapping blocks of the analysis filters, that is, $\boldsymbol{A}_{0}^{T} \cdot \boldsymbol{A}_{1}=\mathbf{0}$, is sufficient to satisfy (22b) since one can choose $\boldsymbol{B}_{i}=\boldsymbol{A}_{i} \cdot\left[\boldsymbol{A}_{0}^{T} \boldsymbol{A}_{0}+\boldsymbol{A}_{1}^{T} \boldsymbol{A}_{1}\right]^{-T}$ and achieve perfect reconstruction. Now, if the normalization equation is also satisfied, that is,

$$
\boldsymbol{A}_{0}^{T} \cdot \boldsymbol{A}_{0}+\boldsymbol{A}_{1}^{T} \cdot \boldsymbol{A}_{1}=\boldsymbol{I}
$$

then obviously, $\boldsymbol{B}_{i}$ has to be chosen equal to $\boldsymbol{A}_{i}$ and we have a paraunitary solution. In that case, the matrix product in (4) is commutative (recall that the system is criti- cally sampled, implying that $\boldsymbol{H}_{p}(z)$ is of size $N$ by $\left.N\right)$ :

$$
\left[\boldsymbol{H}_{p}\left(z^{-1}\right)\right]^{T} \cdot \boldsymbol{H}_{p}(z)=\boldsymbol{H}_{p}(z) \cdot\left[\boldsymbol{H}_{p}\left(z^{-1}\right)\right]^{T}=\boldsymbol{I} .
$$

From (24), it follows that the following two relations hold simultaneously. First, we have that

$$
\begin{array}{r}
\boldsymbol{A}_{0}^{T} \cdot \boldsymbol{A}_{0}+\boldsymbol{A}_{1}^{T} \cdot \boldsymbol{A}_{1}=\boldsymbol{I} \\
\boldsymbol{A}_{0}^{T} \cdot \boldsymbol{A}_{\mathbf{I}}=\mathbf{0}
\end{array}
$$

and then also

$$
\begin{array}{r}
\boldsymbol{A}_{0} \cdot \boldsymbol{A}_{0}^{T}+\boldsymbol{A}_{1} \cdot \boldsymbol{A}_{1}^{T}=\boldsymbol{I} \\
\boldsymbol{A}_{1} \cdot \boldsymbol{A}_{0}^{T}=\mathbf{0} .
\end{array}
$$

Note that (25) and (26) are absolutely equivalent, but that (25a) and (26b) are not equivalent to (25b) and (26a). The orthogonality condition in (25b) means that the $N$ columns of $\boldsymbol{A}_{0}$ are each orthogonal to the $N$ columns of $\boldsymbol{A}_{1}$, while (26b) means the same for the rows of $\boldsymbol{A}_{0}$ and $\boldsymbol{A}_{1}$. The normalization in (26a) means that the $N$ filters form a size $N$ orthonormal basis set.

\section{B. Lapped Orthogonal Transforms (LOT)}

Lapped orthogonal transforms have been introduced by Cassereau [4] and further investigated by Malvar [14]. They are essentially obtained by condition (26) and the additional constraints that the number of channels is even and that the filters have linear phase $(N / 2$ symmetric and $N / 2$ antisymmetric filters ). In that sense, LOT's are a subclass of paraunitary solutions (linear phase and length constraint). Note that condition $\mathrm{Cl}$ together with $\boldsymbol{T}_{d}=\boldsymbol{I}$ is the extension of the LOT concept to arbitrary filter lengths.

The orthogonality constraint means that $\boldsymbol{A}_{0}$ and $\boldsymbol{A}_{1}$ span orthogonal subspaces of the size $N$ vector space. Cassereau has shown [4] that, when the linear phase constraint is added, then $\boldsymbol{A}_{0}$ and $\boldsymbol{A}_{1}$ span each a subspace of size $N / 2$. Based on these considerations, design techniques have been developed [4], [14]. However, the techniques do not generalize well for lengths greater than $2 N$ (for example, some dependencies which are linear for $L_{a}=$ $2 \mathrm{~N}$ become nonlinear for longer filters). From a computational point of view, Malvar has shown [14] that the LOT can be based on a fast transform (a DCT typically), thus making the LOT computationally very efficient (two fast transforms and $N / 2$ rotations as a typical case).

It is interesting to note that two techniques that have been developed independently, namely, perfect reconstruction FIR filter banks and lapped orthogonal transforms, lead to the same solution characterized by paraunitary (in $z$-transform domain) or unitary (in time-domain) matrices.

\section{Modulated Filter Banks with Perfect Reconstruction}

Pseudo-QMF filters have been proposed as an extension to $N$ channels of the classical two-band QMF filters [22], [28], [24], [5], [6], [15]. Pseudo-QMF analysis/ synthesis systems achieve, in general, only the cancella- 
tion of the main aliasing term. However, when the filter length is restricted to $L_{a}=2 N$, they can achieve perfect reconstruction under certain conditions. The main advantages of pseudo-QMF filters are their low computational complexity as well as the fact that the window function can be tuned to satisfy additional design constraints while maintaining the exact reconstruction property.

Assume that $N$ is even and that critical subsampling is used. Then the $i$ th analysis and synthesis filters are obtained by modulating a real prototype filter evenly over the frequency spectrum. While the modulating frequencies are easily obtained from the fact that the whole spectrum has to be covered with real filters, the selection of a phase term for the analysis and the synthesis is more delicate. A family of pseudo-QMF filter banks that achieves main aliasing cancellation has been designed in [15] and is of the form

$$
\begin{aligned}
h_{k}(n)= & h_{p r}(n) \cdot \cos \left(\frac{2 \pi(2 k+1)}{4 N}\right. \\
& \left.\cdot\left(n-\left(\frac{L-1}{2}\right)\right)+\phi_{k}\right)
\end{aligned}
$$

for the analysis filters $\left(h_{p r}(n)\right.$ is the impulse response of the prototype filter). In the general case, the main aliasing term is cancelled for the value of the phase:

$$
\phi_{k}=\frac{\pi}{4}+k \frac{\pi}{2}
$$

and it can be shown that this property also holds for any " "nonsingular'" value of the prototype filter. The synthesis filters have the same modulation, but with a negative phase term equal to $-\pi / 4-k \pi / 2$.

In the case $L_{a}=2 N$ and assuming that $h_{p r}(n)=$ $1 / \sqrt{N}, n=0, \cdots, 2 N-1$, it can be verified that (21) and (25) hold, leading to a paraunitary solution [class 3$)$ ]. The matrices $\boldsymbol{A}_{0}$ and $\boldsymbol{A}_{1}$ of the unwindowed filter bank ( $h_{p r}$ $=1 / \sqrt{N}, n=0, \cdots, 2 N-1)$ satisfy the relation

$$
\begin{aligned}
\boldsymbol{A}_{0}^{T} \cdot \boldsymbol{A}_{1} & =0 \\
\boldsymbol{A}_{0}^{T} \cdot \boldsymbol{A}_{0}+\boldsymbol{A}_{1}^{T} \cdot \boldsymbol{A}_{1} & =\boldsymbol{I} \\
\boldsymbol{A}_{0}^{T} \cdot \boldsymbol{A}_{0} & =\frac{1}{2} \cdot(\boldsymbol{I}+\boldsymbol{J}) \\
\boldsymbol{A}_{1}^{T} \cdot \boldsymbol{A}_{1} & =\frac{1}{2} \cdot(\boldsymbol{I}-\boldsymbol{J}) .
\end{aligned}
$$

While the two first relations are common to any $L_{a}=2 \mathrm{~N}$ class 3) solution, relations (29c) and (29d) are particular to the modulated filter bank. The property that a filter bank derived by a modulation of a window function can lead to exact reconstruction in the case $L_{a}=2 N$ had also been recognized by Princen et al. [25], [26] in a slightly different context.

The fact that a window (prototype filter) can be applied to the pseudo-QMF filter bank while preserving alias cancellation leads us to investigate for the case $L_{a}=2 \mathrm{~N}$ what condition the window $h_{p r}(n)$ must satisfy for exact re- construction and paraunitariness to be preserved. Assume that a symmetric window function or prototype filter is used. The new matrices $\boldsymbol{A}_{0}^{\prime}$ and $\boldsymbol{A}_{1}^{\prime}$ are given by

$$
\begin{aligned}
& \boldsymbol{A}_{0}^{\prime}=\boldsymbol{A}_{0} \cdot \boldsymbol{W} \\
& \boldsymbol{A}_{\mathrm{l}}^{\prime}=\boldsymbol{A}_{\mathrm{l}} \cdot \boldsymbol{W}^{\prime}
\end{aligned}
$$

where $\boldsymbol{W}$ is a diagonal windowing matrix given by

$$
\boldsymbol{W}=\left(\begin{array}{cccc}
h_{p r}(0) & 0 & \cdots & \ldots \\
0 & h_{p r}(1) & 0 & \ldots \\
\ldots & \ldots & \ddots & 0 \\
\ldots & \ldots & 0 & h_{p r}(N-1)
\end{array}\right)
$$

and $\boldsymbol{W}^{\prime}=\boldsymbol{J} \cdot \boldsymbol{W} \cdot \boldsymbol{J}$ has the diagonal element of $\boldsymbol{W}$ in reverse order. We can readily verify that the condition $\boldsymbol{A}_{0}^{\prime T} \cdot \boldsymbol{A}_{1}^{\prime}=\mathbf{0}$ is verified since

$$
\boldsymbol{A}_{0}^{\prime T} \cdot \boldsymbol{A}_{1}^{\prime}=\boldsymbol{W} \cdot \boldsymbol{A}_{0}^{T} \cdot \boldsymbol{A}_{1} \cdot \boldsymbol{J} \cdot \boldsymbol{W} \cdot \boldsymbol{J}=\mathbf{0}
$$

follows from (29a). If we compute the product $\boldsymbol{A}_{0}^{\prime T} \cdot \boldsymbol{A}_{0}^{\prime}$ $+\boldsymbol{A}_{1}^{\prime T} \cdot \boldsymbol{A}_{1}^{\prime}$ and take into account (29c) and (29d), we find that

$$
\boldsymbol{A}_{0}^{\prime T} \cdot \boldsymbol{A}_{0}^{\prime}+\boldsymbol{A}_{1}^{\prime T} \cdot \boldsymbol{A}_{1}^{\prime}=\frac{1}{2}\left(\boldsymbol{W}^{2}+\boldsymbol{W}^{\prime 2}\right)
$$

The matrix $\boldsymbol{W}^{2}+\boldsymbol{W}^{\prime 2}$ is a diagonal matrix with the $i$ th diagonal element of the form

$$
h_{p r}^{2}(i)+h_{p r}^{2}(N-1-i) .
$$

This allows us to state the following result.

Result: The windowed modulated filter bank allows exact reconstruction in the case $L_{a}=2 N$ if and only if the matrix $\boldsymbol{W}^{2}+\boldsymbol{W}^{\prime 2}$ is nonsingular; furthermore, if the window meets the condition

$$
h_{p r}^{2}(i)+h_{p r}^{2}(N-1-i)=2
$$

then the solution is paraunitary. This result was also found by Princen et al. [25], [26] for another modulated filter bank, hinting that the solution above would work as well.

Some discussion of the windowed pseudo-QMF scheme seems appropriate; first, we note that windowing will never destroy the orthogonality of overlapping blocks (the columns of $\boldsymbol{A}_{0}$ and $\boldsymbol{A}_{1}$ are simply weighted by the window function, thus conserving their mutual orthogonality). Note that this result holds true for filters of arbitrary length. Therefore, exact reconstruction with filters of the same length is conserved over windowing. The degree of freedom introduced by the window allows one to trade off frequency-domain and time-domain properties of the filter bank, thus optimizing the design for a given application.

It is the particular form of the products $\boldsymbol{A}_{i}^{T} \cdot \boldsymbol{A}_{i}(29 \mathrm{c})$ and $(29 \mathrm{~d})$ that provides the "window independence." For the sake of illustration, we indicate an example on how to generate matrices $\boldsymbol{A}_{i}$ that will satisfy (29c). Assume an orthogonal basis of size $N$ given by the vectors 
$V_{0} \quad V_{1} \cdots V_{N-1}$; then choose

$$
\begin{aligned}
& \boldsymbol{A}_{0}=\left[\begin{array}{llllllll}
V_{0} & V_{1} & \cdots & V_{(N / 2)-1} & V_{(N / 2)-1} & \cdots & V_{1} & V_{0}
\end{array}\right] \\
& \boldsymbol{A}_{1}=\left[\begin{array}{lllll}
V_{N / 2} & V_{(N / 2)+1} & \cdots & V_{N-1} & -V_{N-1}
\end{array} \cdots\right. \\
& \left.-V_{(N / 2)+1}-V_{N / 2}\right] \text {. }
\end{aligned}
$$

Then $(29 \mathrm{c})$ and $(29 \mathrm{~d})$ are automatically satisfied. The pseudo-QMF filter bank is a particular case of (36). Unfortunately, such a technique to design the $L_{a}=2 \mathrm{~N}$ filter bank excludes linear phase solutions. The condition on the window given by (35) is quite important; if it does not hold, the synthesis filter bank will be given by

$$
\boldsymbol{B}_{i}=\boldsymbol{A}_{i} \cdot\left(\boldsymbol{W}^{2}+\boldsymbol{W}^{\prime 2}\right)^{-1} \text {. }
$$

The inverse which has to be applied at the synthesis might deemphasize any benefits introduced by the window. This concludes the discussion of perfect reconstruction modulated filter banks. The main purpose of this section was to demonstrate their existence, which was doubted previously because filter banks based on complex modulation (DFT) do not allow perfect FIR reconstruction [46], [34]. Also, the effect of windowing was investigated and a simple condition was shown to preserve the paraunitary property.

\section{Solutions Based on Product Forms}

In this section, we will consider solutions to the perfect reconstruction filter bank problem that will be written in product form [see (20)] and that will meet certain additional constraints. More specifically, matrices of polyphase components will be derived that lead to analysis and synthesis filters having linear phase and/or modulation relations among themselves. The technique that will be used forms cascades of elementary matrices so that the resulting polyphase matrix satisfies the desired properties. Note that the elementary matrices are chosen independently of one another. This cascade generation is only one of many possible approaches (it can actually be overconstrained in some cases), but it is interesting both for synthesis and implementation purposes. Note that the two-channel linear phase case that will be shown below was also independently derived by Nguyen and Vaidyanathan [21].

\section{A. The Two-Channel Case, $N=2$}

Let us first recall the paraunitary solution proposed by Vaidyanathan [36], [37], [39]. In that case, the polyphase matrix $\boldsymbol{H}_{p}(z)$ of the analysis filters can be written as

$$
\boldsymbol{H}_{p}(z)=\left(\begin{array}{cc}
1 & \alpha_{0} \\
-\alpha_{0} & 1
\end{array}\right) \cdot \prod_{k=1}^{K-1}\left(\begin{array}{cc}
1 & 0 \\
0 & z^{-1}
\end{array}\right)\left(\begin{array}{cc}
1 & \alpha_{k} \\
-\alpha_{k} & 1
\end{array}\right) .
$$

This leads to nonlinear phase filters of length $L=2 K$ which are like the ones proposed (in noncascaded form) in [29], [30], [16]. The impulse responses of the two filters are related in such a way $\left[h_{1}(n)=(-1)^{n} \cdot h_{0}(L-\right.$ $n-1)]$ that linear phase solutions are excluded (except in the trivial case of length 2 filters). Note that (38) is a denormalized version since the "rotation" matrices do not have unit length vectors. This can be taken care of at the reconstruction, with the polyphase matrix of the synthesis filters chosen as [36]

$$
\begin{aligned}
\boldsymbol{G}_{p}(z)= & \frac{1}{1+\alpha_{0}^{2}} \cdot\left(\begin{array}{cc}
1 & \alpha_{0} \\
-\alpha_{0} & 1
\end{array}\right) \cdot \prod_{k=1}^{K-1}\left(\begin{array}{ll}
z^{-1} & 0 \\
0 & 1
\end{array}\right) \\
& \cdot\left(\begin{array}{cc}
1 & \alpha_{k} \\
-\alpha_{k} & 1
\end{array}\right) \cdot \frac{1}{1+\alpha_{k}^{2}}
\end{aligned}
$$

Note that a normalized version would use a factor $1 / \sqrt{1+\alpha^{2}}$ both in (38) and (39) (leading to elementary matrices which are rotations). The product $\left[\boldsymbol{G}_{p}(z)\right]^{T}$. $\boldsymbol{H}_{p}(z)$ [see (3)] is equal to

$$
\left[\boldsymbol{G}_{p}(z)\right]^{T} \cdot \boldsymbol{H}_{p}(z)=z^{-(K-1)} \cdot \boldsymbol{I}
$$

and perfect reconstruction is achieved with a delay of $L$ - 1 samples. A nice property of this solution is that it is complete [39], that is, all paraunitary solutions can be generated with this structure (a fact related to the factorization of paraunitary matrices [1]).

A linear phase solution is obtained if the two filters $H_{0}(z)$ and $H_{1}(z)$ obtained from the polyphase filter matrix $\boldsymbol{H}_{p}(z)$

$$
\left(\begin{array}{l}
H_{0}(z) \\
H_{1}(z)
\end{array}\right)=\left(\begin{array}{ll}
H_{0,0}\left(z^{2}\right) & H_{1,0}\left(z^{2}\right) \\
H_{1,0}\left(z^{2}\right) & H_{1,1}\left(z^{2}\right)
\end{array}\right) \cdot\left(\begin{array}{c}
1 \\
z^{-1}
\end{array}\right)
$$

are symmetric and antisymmetric, respectively. Assume that the two filters are of the same length, in which case the length has to be even [46]. Then, $\boldsymbol{H}_{p}(z)$ has to satisfy the following "linear phase" test:

$$
\left(\begin{array}{rr}
1 & 0 \\
0 & -1
\end{array}\right) \cdot\left[z^{-k} \cdot \boldsymbol{H}_{p}\left(z^{-1}\right)\right] \cdot\left(\begin{array}{ll}
0 & 1 \\
1 & 0
\end{array}\right)=\boldsymbol{H}_{p}(z)
$$

where $k$ is the highest degree in $\boldsymbol{H}_{p}(z)$. We will now develop a cascade form to obtain linear phase filters of any even length and which guarantee perfect reconstruction:

1) assume $\boldsymbol{H}_{p}(z)$ satisfies (42)

2) then $\boldsymbol{H}_{p}^{\prime}(z)$ given by

$$
\boldsymbol{H}_{p}^{\prime}(z)=\boldsymbol{H}_{p}(z) \cdot\left(\begin{array}{cc}
1 & 0 \\
0 & z^{-1}
\end{array}\right) \cdot\left(\begin{array}{cc}
1 & \alpha \\
\alpha & 1
\end{array}\right)
$$

satisfies (42) as well.

The proof is straightforward by replacing (43) into (42) and verifying that it holds indeed. Note that for $L=2$, the two only possible filters are given by $H_{0}(z)=1+$ $z^{-1}$ and $H_{1}(z)=1-z^{-1}$ (or scaled versions thereof), and therefore, a possible way to obtain length $L=2 \mathrm{~K}$ linear phase perfect reconstruction filters is by writing $\boldsymbol{H}_{p}(z)$ as [49]

$$
\boldsymbol{H}_{p}(z)=\left(\begin{array}{cc}
1 & 1 \\
1 & -1
\end{array}\right) \cdot \prod_{k=1}^{K-1}\left(\begin{array}{cc}
1 & 0 \\
0 & z^{-1}
\end{array}\right)\left(\begin{array}{cc}
1 & \alpha_{k} \\
\alpha_{k} & 1
\end{array}\right)
$$




$$
\begin{aligned}
& \text { and } \boldsymbol{G}_{p}(z) \text { as } \\
& \begin{aligned}
\boldsymbol{G}_{p}(z)= & \frac{1}{2} \cdot\left(\begin{array}{rr}
1 & 1 \\
1 & -1
\end{array}\right) \cdot \prod_{k=1}^{K-1}\left(\begin{array}{ll}
z^{-1} & 0 \\
0 & 1
\end{array}\right)\left(\begin{array}{rr}
1 & -\alpha_{k} \\
-\alpha_{k} & 1
\end{array}\right) \\
& \cdot \frac{1}{1-\alpha_{k}^{2}} .
\end{aligned}
\end{aligned}
$$

Therefore, the product (3) is satisfied and equal to (40). Note that while this is not a paraunitary solution (and condition $\mathrm{Cl}$ is not verified in general), the synthesis filters are simply related to the analysis filters by a modulation with $(-1)^{n}$ :

$$
G_{0}(z)=H_{1}(-z) \quad G_{1}(z)=-H_{0}(-z) .
$$

The first few filters obtained from (44) are given in $\mathrm{Ta}$ ble II. Note that length $2 K$ filters are defined by $K-1$ free parameters.

Note that if one wants odd length filters (one of length $2 N+1$ and the other of length $2 N-1$ ), a zero can be exchanged between $H_{0}(z)$ and $H_{1}(-z)$ since this will not alter the perfect reconstruction property. The resulting filters are now both symmetric and of (different) odd lengths. This technique has been used in [13] to derive good and efficient filters for subband coding of images. This method is only a particular case of a more general structure for odd length symmetric filters which is described in Appen$\operatorname{dix} A$. It is shown there that two symmetric and linear phase filters of length $2 K+1$ and $2 K+3$ can be generated with $2 K$ free parameters (that is, about twice as many as in the even length case). However, experience with such designs shows that the additional freedom cannot be really used [21], [42].

Having derived possible generic structures for linear phase filters, one may want to know their generality. Because $\boldsymbol{H}_{p}(z)$ is not paraunitary, there is no factorization theorem that can be used (at least directly). Appendix B shows that the factorization given in (44) for even length filters is complete for lengths smaller than 8 . For longer filters, there exist perfect reconstruction filter banks that cannot be written in the factorized form (44) (see Appendix B). These "singular" cases seem isolated and can be approximated. They seem, however, of little practical importance.

Finally, in the two-channel case, note that while previous techniques existed to find linear phase perfect reconstruction filters (like the complementary filter method or the factorization method [47]), the above form structurally guarantees perfect reconstruction (similarly to the paraunitary case [39]). Note also that the factors $\alpha_{i}$ can be chosen so as to minimize the resulting hardware complexity of an implementation. This was used in [13] to

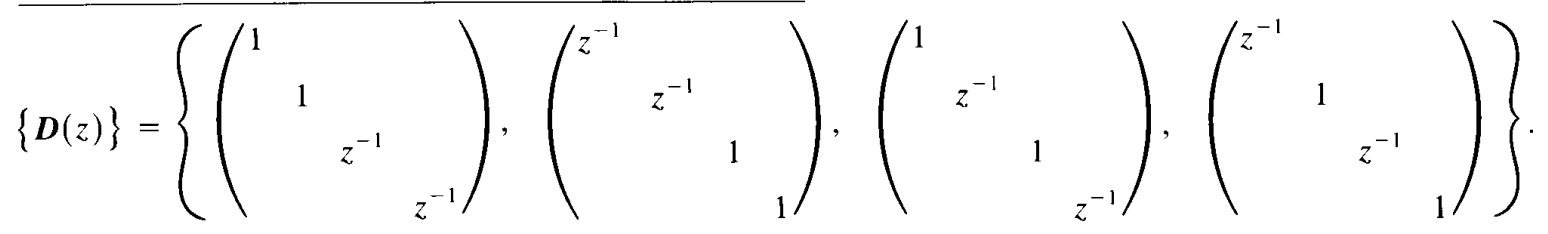

\begin{tabular}{|c|c|c|}
\hline $\mathrm{L}$ & $\begin{array}{l}\text { Scaling } \\
\text { factor }\end{array}$ & Analysis \& synthesis Filters \\
\hline 2 & 2 & $\begin{array}{l}H_{0}(z)=1+z^{-1} \\
H_{1}(z)=1-z^{-1}\end{array}$ \\
\hline 4 & $2\left(1-\alpha_{1}^{2}\right)$ & $\begin{array}{l}H_{0}(z)=1+\alpha z^{-1}+\alpha z^{-2}+z^{-3} \\
H_{1}(z)=1+\alpha z^{-1}-\alpha z^{-2}-z^{-3}\end{array}$ \\
\hline 6 & $2\left(1-\alpha_{1}^{2}\right)\left(1-\alpha_{2}^{2}\right)$ & $\begin{array}{c}H_{0}(z)=1+\alpha_{2} z^{-1}+\left(\alpha_{1}+\alpha_{1} \alpha_{2}\right) z^{-2}+\left(\alpha_{1}+\alpha_{1} \alpha_{2}\right) z^{-3}+\alpha_{2} z^{-4}+z^{-5} \\
H_{1}(z)=1+\alpha_{2} z^{-1}+\left(-\alpha_{1}+\alpha_{1} \alpha_{2}\right) z^{-2}+\left(\alpha_{1}-\alpha_{1} \alpha_{2}\right) z^{-3}-\alpha_{2} z^{-4}-z^{-5}\end{array}$ \\
\hline
\end{tabular}

Linfar Phasf Perfect Reconstruction Filters Obtained from the. Cascade Form (44). The Scaling Factor is the Term that has to be Divided out BetweEn ANALYSIS AND Synthesis. The SyNTHESIS FILTERS ARE GIVEN BY $G_{0}(z)=H_{1}(-z)$ AND $G_{1}(z)=-H_{0}(-z)$

generate very simple filters for high-speed image processing.

\section{B. Linear Phase Solutions, $N>2$}

Similar to (42), a polyphase matrix that leads to linear phase filters has to satisfy (we assume $N$ even, and that the first $N / 2$ filters are symmetric while the last $N / 2$ are antisymmetric)

$$
\left(\begin{array}{rr}
\boldsymbol{I} & 0 \\
0 & -\boldsymbol{I}
\end{array}\right) \cdot\left[z^{-k} \cdot \boldsymbol{H}_{p}\left(z^{-1}\right)\right] \cdot \boldsymbol{J}=\boldsymbol{H}_{p}(z)
$$

where $\boldsymbol{I}$ is the identity matrix of size $N / 2$ and $\boldsymbol{J}$ is the antidiagonal matrix of size $N$. In order to generate polyphase matrices that satisfy (47), we write a new polyphase matrix $\boldsymbol{H}_{p}^{\prime}(z)$ as

$$
\boldsymbol{H}_{p}^{\prime}(z)=\boldsymbol{H}_{p}(z) \cdot \boldsymbol{D}(z) \cdot \boldsymbol{R}
$$

where $\boldsymbol{H}_{p}(z)$ satisfies $(47), \boldsymbol{D}(z)$ is a diagonal matrix of delays, and $\boldsymbol{R}$ is a unitary matrix. In that case, the following two conditions are necessary and sufficient for $\boldsymbol{H}_{p}^{\prime}(z)$ to satisfy (47) as well:

$$
\begin{aligned}
z^{-l} \cdot \boldsymbol{J} \cdot \boldsymbol{D}\left(z^{-1}\right) \cdot \boldsymbol{J} & =\boldsymbol{D}(z) \\
\boldsymbol{J} \cdot \boldsymbol{R} \cdot \boldsymbol{J} & =\boldsymbol{R}
\end{aligned}
$$

where $l$ in (49) is the highest degree of $z^{-1}$ present in $D(z)$. A matrix $R$ that satisfies (50) is called persymmetric [2].

As a starting matrix in (48), one can take any unitary transform that has $N / 2$ symmetric and $N / 2$ antisymmetric vectors such as, for example, the Walsh-Hadamard ( $N=2^{m}$ ) or the discrete cosine transform. There is obviously a large set of possible diagonal matrices of delays that satisfy (49). For example, in the case $N=4$, we list the four possible matrices $D(z)$ : 
Note that these matrices are paraunitary, like all diagonal matrices of delays. Also, antidiagonal matrices with the same elements as in (51) would satisfy (49) as well, but lead basically to the same solution within a permutation.

The condition (50) on the matrix $\boldsymbol{R}$ is even more relaxed. For example, all symmetric Toeplitz matrices satisfy (50). A closer look shows that matrices satisfying (50) are of the form

$$
\boldsymbol{R}=\left(\begin{array}{ll}
\boldsymbol{M}_{0} & \boldsymbol{M}_{1} \\
\boldsymbol{J} \boldsymbol{M}_{1} J & J M_{0} J
\end{array}\right)
$$

where $\boldsymbol{M}_{0}$ and $\boldsymbol{M}_{1}$ are size $N / 2$ by $N / 2$ matrices, and $\boldsymbol{J}$ is the antidiagonal matrix of size $N / 2$. One can verify that (50) is satisfied since

$$
\left(\begin{array}{ll}
0 & J \\
J & 0
\end{array}\right)\left(\begin{array}{ll}
M_{0} & M_{1} \\
J M_{1} J & J M_{0} J
\end{array}\right)\left(\begin{array}{ll}
0 & J \\
J & 0
\end{array}\right)=\left(\begin{array}{ll}
M_{0} & M_{1} \\
J M_{1} J & J M_{0} J
\end{array}\right)
$$

where we used the fact that $\boldsymbol{J}^{2}=\boldsymbol{I}$. If $\boldsymbol{R}$ is required to be unitary, that is (assuming real coefficients),

$$
\boldsymbol{R}^{T} \cdot \boldsymbol{R}=\boldsymbol{R} \cdot \boldsymbol{R}^{T}=\boldsymbol{I}
$$

then it can be verified that the matrices $\boldsymbol{M}_{0}$ and $\boldsymbol{M}_{1}$ have to satisfy

$$
\begin{array}{r}
\boldsymbol{M}_{0} \cdot \boldsymbol{M}_{0}^{T}+\boldsymbol{M}_{1} \cdot \boldsymbol{M}_{1}^{T}=\boldsymbol{I} \\
\boldsymbol{M}_{1} \cdot \boldsymbol{J} \cdot \boldsymbol{M}_{0}^{T}+\boldsymbol{M}_{0} \cdot \boldsymbol{J} \cdot \boldsymbol{M}_{1}^{T}=\mathbf{0}
\end{array}
$$

which corresponds to $\boldsymbol{R} \cdot \boldsymbol{R}^{T}=\boldsymbol{I}$. Since the product (54) is commutative, $\boldsymbol{R}^{T} \cdot \boldsymbol{R}=\boldsymbol{I}$ leads to another, but equivalent, set of conditions. Note that $(55 \mathrm{a})$ is the usual orthonormality of the first $N / 2$ rows of $\boldsymbol{R}$, while (55b) captures the inherent "symmetry" of $\boldsymbol{R}$ that is required in order to meet (50). A simple example, for $N=4$, would be [17]

$$
\begin{aligned}
\boldsymbol{R}= & \left(\begin{array}{llll}
\cos \left(\alpha_{1}\right) & -\sin \left(\alpha_{1}\right) & 0 & 0 \\
\sin \left(\alpha_{1}\right) & \cos \left(\alpha_{1}\right) & 0 & 0 \\
0 & 0 & \cos \left(\alpha_{1}\right) & \sin \left(\alpha_{1}\right) \\
0 & 0 & -\sin \left(\alpha_{1}\right) & \cos \left(\alpha_{1}\right)
\end{array}\right) \\
& \left(\begin{array}{llll}
\cos \left(\alpha_{2}\right) & 0 & -\sin \left(\alpha_{2}\right) & 0 \\
0 & \cos \left(\alpha_{2}\right) & 0 & \sin \left(\alpha_{2}\right) \\
\sin \left(\alpha_{2}\right) & 0 & \cos \left(\alpha_{2}\right) & 0 \\
0 & -\sin \left(\alpha_{2}\right) & 0 & \cos \left(\alpha_{2}\right)
\end{array}\right)
\end{aligned}
$$

which satisfies (50) or (55) as can be checked (we used the fact that the product of two persymmetric matrices is persymmetric).

Instead of a postmultiplication in (48), one may want a premultiplication. It turns out that no delay matrix $D(z)$ (except trivial ones) will satisfy (47), but that any matrix $\boldsymbol{R}$ which is block diagonal with blocks of size $N / 2$ by

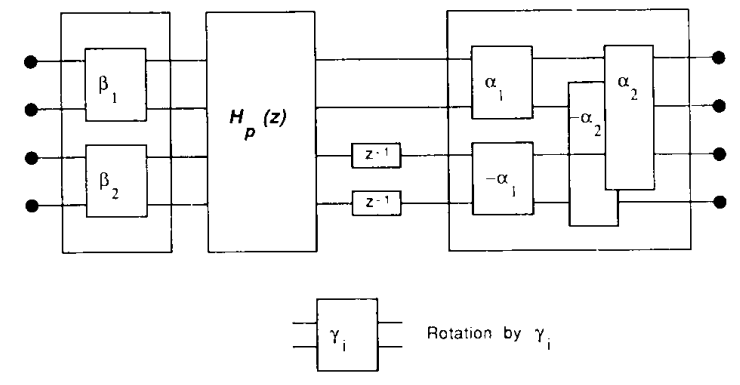

Fig. 4. Generation of a new polyphase matrix $\boldsymbol{H}_{p}^{\prime}(z)$ from pre- and postmultiplication of an initial solution $\boldsymbol{H}_{p}(z)$. If $\boldsymbol{H}_{p}(z)$ is paraunitary and corresponds to linear phase filters, so will $H_{p}^{\prime}(z)$.

$N / 2$ will. Therefore, any solution $\boldsymbol{H}_{p}(z)$ can be modified by a premultiplication with such a block-diagonal matrix. This is similar to techniques used in "lapped orthogonal transforms" (LOT) [14] where the filters are, however, restricted to $L=2 N$.

Fig. 4 shows an example for $N=4$. Starting with a polyphase matrix $\boldsymbol{H}_{p}(z)$ that satisfies (47) and is paraunitary, one obtains a new polyphase matrix $\boldsymbol{H}_{p}^{\prime}(z)$ which satisfies (47) as well and is also paraunitary. This is achieved by a premultiplication with a unitary block-diagonal matrix $\boldsymbol{R}^{\prime \prime}$ and postmultiplication by a delay matrix from (51) as well as by a rotation matrix $\boldsymbol{R}^{\prime}$ as in (56).

Therefore, for $N>2$, we showed that paraunitary solutions that lead to linear phase filters exist, and this for arbitrary length filters. The question of completeness of the structures needs, however, more investigation.

\section{Filters Satisfying Frequency Symmetry Constraints}

Assume that in the filter bank, filters with index $i$ and $N-i(i=0 \cdots N / 2-1)$ are related by a modulation with $(-1)^{n}$, that is, in the $z$-transform domain

$$
H_{N-i-1}(z)=H_{i}(-z) \quad i=0 \cdots N / 2-1 \text {. }
$$

For $N=2$, this is the classical QMF case. For $N>2$, this case has also been considered in [38] and we derive a cascade structure for it. The condition in (57) forces symmetries in the polyphase matrix $\boldsymbol{H}_{p}(z)$ since even numbered polyphase components of $H_{i}(z)$ and $H_{N-i-1}(z)$ are equal, while odd numbered ones have opposite sign. Define a matrix $T$ of size $N$ by $N$ as

$$
\boldsymbol{T}=\left(\begin{array}{cccccccr}
1 & 0 & 0 & \cdots & \cdots & 0 & 0 & 1 \\
1 & 0 & 0 & \cdots & \cdots & 0 & 0 & -1 \\
0 & 1 & 0 & \cdots & \cdots & 0 & 1 & 0 \\
\cdot & \cdot & . & \cdots & \cdots & . & \cdot & \cdot \\
0 & \cdots & 0 & 1 & -1 & 0 & \cdots & 0
\end{array}\right) .
$$

Now, because of the symmetry in (57), we can verify that

$$
\begin{aligned}
{\left[\boldsymbol{T} \cdot \boldsymbol{H}_{p}(z)\right]_{i, j} } & =2 H_{i, j}(z), \quad i+j \text { even } \\
& =0, \quad i+j \text { odd }
\end{aligned}
$$


where $i$ and $j$ are the row and column indexes with the numbering starting from 0 . As in previous sections, we would like to be able to generate polyphase matrices satisfying (59). More precisely, assume we have a matrix $\boldsymbol{H}_{p}(z)$ that satisfies (59), and that we obtain a matrix $\boldsymbol{H}_{p}^{\prime}(z)$ by pre- or postmultiplying $\boldsymbol{H}_{p}(z)$ with a delay or "rotation" matrix; then how should we choose these so that $\boldsymbol{H}_{p}^{\prime}(z)$ satisfies $(59)$ as well? Note that the delay and "rotation" matrix should be independent of $\boldsymbol{H}_{p}(z)$. Let us consider the following four cases.

1) Premultiplication by a Delay Matrix $D(z)$ : It is easy to verify that $D(z)$ (which is diagonal by assumption) has to meet

$$
[\boldsymbol{D}(z)]_{i i}=[\boldsymbol{D}(z)]_{N-i-1, N-i-1} .
$$

2) Premultiplication by a Rotation Matrix $\boldsymbol{R}$ : We call this a "rotation" matrix because it will be, in general, chosen as a unitary matrix (but not necessarily). Then the rows of $\boldsymbol{R}$ have to be related by

$$
\operatorname{row}_{N-i-1}=\operatorname{row}_{i} \cdot \boldsymbol{J}
$$

so that $\boldsymbol{H}_{p}^{\prime}(z)$ meets $(50)$. Note that (61) means that $\boldsymbol{R}$ is persymmetric.

3) Postmultiplication by a Delay Matrix $D(z)$ : No condition is necessary since as long as $D(z)$ is diagonal, $\boldsymbol{H}_{p}^{\prime}(z)$ will meet (59).

4) Postmultiplication by a Rotation Matrix R: In that case, it is necessary and sufficient that

$$
\boldsymbol{R}_{i, j}=0, \quad i+j \text { odd }
$$

and $H_{p}^{\prime}(z)$ will meet (59) as well.

Note that 1)-4) give conditions so that (59) is met recursively, and this is independently of the previous terms in the cascade.

An example of recursive generation for the case $N=4$ is given in Fig. 5. Premultiplication of the initial solution $\boldsymbol{H}_{p}(z)$ is done by a matrix satisfying (60) and a rotation matrix that is persymmetric. Postmultiplication is by an arbitrary delay matrix and a rotation matrix satisfying (62). The new polyphase matrix $\boldsymbol{H}_{p}^{\prime}(z)$ then satisfies (59) by construction.

Now, if the matrices $D(z)$ and $\boldsymbol{R}$ meet additional constraints, like the ones required for linear phase or paraunitariness, then the resulting polyphase matrix will yield linear phase filters or be paraunitary [on top of leading to the frequency symmetry given by (57)]. As a simple example, look at the following postmultiplication matrix:

$$
\boldsymbol{R}_{\alpha}=\left(\begin{array}{llll}
\cos (\alpha) & 0 & -\sin (\alpha) & 0 \\
0 & \cos (\alpha) & 0 & \sin (\alpha) \\
\sin (\alpha) & 0 & \cos (\alpha) & 0 \\
0 & -\sin (\alpha) & 0 & \cos (\alpha)
\end{array}\right)
$$

This matrix satisfies (50) (linear phase constraint), (54) (it is a unitary matrix), and (62) (frequency symmetry).

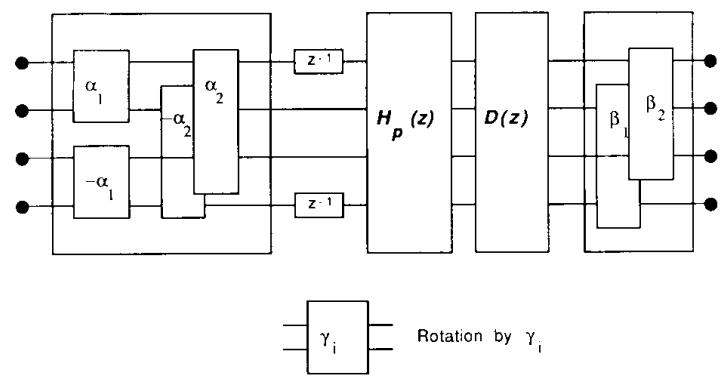

Fig. 5. Generation of a new polyphase matrix $\boldsymbol{H}_{n}^{\prime}(z)$ from pre- and postmultiplication of an initial solution $\boldsymbol{H}_{p}(z)$. If $\boldsymbol{H}_{p}(z)$ satisfies $(59 \mathrm{a})$ and is paraunitary, so will $\boldsymbol{H}_{p}^{\prime}(z)$ by construction.

When used together with delay matrices as in (51) as well as a starting matrix that satisfies (59) (like the WalshHadamard or the discrete cosine transform matrix of size 4 by 4 ), this rotation matrix will lead to a perfect reconstruction, linear phase filter bank with frequency symmetry $(N=4$, arbitrary filter length, and identical analysis and synthesis filters). Of course, (63) is very constrained, and does not lead to very interesting filters. The point was to show that solutions to such heavily constrained filter banks exist and can be constructed as cascades of simple elementary blocks.

\section{Computational Complexity}

Because of their special structure, the filter banks introduced in this paper have, in general, low computational complexity.

\section{A. Two-Channel Case}

Let us first review the paraunitary case [see (38)] where the computational blocks are 2 by 2 rotation matrices [39]. Since a rotation matrix can always be written as [23], [2] [with $a=\cos (\alpha)$ and $b=\sin (\alpha)$ ]

$$
\begin{aligned}
& \left(\begin{array}{rr}
a & -b \\
b & a
\end{array}\right)=\left(\begin{array}{rrr}
1 & -1 & 0 \\
1 & 0 & 1
\end{array}\right) \\
& \cdot\left(\begin{array}{ccc}
a & & \\
& a+b & \\
& & b-a
\end{array}\right) \cdot\left(\begin{array}{ll}
1 & 1 \\
0 & 1 \\
1 & 0
\end{array}\right)
\end{aligned}
$$

it takes three multiplications per rotation. A bank with two filters of length $L=2 K$ has $K$ such blocks and two input values produce two new output values (one in each channel); thus, the computational complexity of a paraunitary two-channel filter bank is $(3 K / 2)$ multiplications per input sample. This was already noted in [11] without using factorization. Now, if one uses denormalized blocks (for example, dividing (64) by $a$ ), then each block takes only two multiplications, and one multiplication is required at the end in each channel in order to renormalize the output. The computational complexity per input sample is therefore equal to $(K+1)$. This result was first noted in [10].

Let us consider the linear phase case next [see (44)]. 
Each computational block can be written as

$$
\begin{aligned}
& \frac{1}{\sqrt{1-a^{2}}}\left(\begin{array}{cc}
1 & a \\
a & 1
\end{array}\right) \\
& =\left(\begin{array}{rr}
1 & 1 \\
1 & -1
\end{array}\right) \cdot\left(\begin{array}{c}
\frac{1+a}{2 \sqrt{1-a^{2}}} \\
\frac{1-a}{2 \sqrt{1-a^{2}}}
\end{array}\right) \\
& \quad \cdot\left(\begin{array}{rr}
1 & 1 \\
1 & -1
\end{array}\right), \quad|a|<1 .
\end{aligned}
$$

The factor $1 / \sqrt{1-a^{2}}$ was used to "normalize" the matrix (in a loose sense since the matrix is not orthogonal). If $|a|>1$, then one can use $1 / \sqrt{a^{2}-1}$ and $-1 / \sqrt{a^{2}-1}$ as a "normalization" factor at the analysis and synthesis, respectively.

Since a block written as (65) requires two multiplications, and there are $K-1$ blocks, the multiplicative complexity of a length $L=2 K$ filter bank is $(K-1)$ multiplications per input sample. Now, if $(65)$ is "denormalized," that is,

$$
\begin{aligned}
\frac{2}{1+a}\left(\begin{array}{ll}
1 & a \\
a & 1
\end{array}\right)= & \left(\begin{array}{rr}
1 & 1 \\
1 & -1
\end{array}\right) \cdot\left(\begin{array}{ll}
1 & 1-a \\
1+a
\end{array}\right) \\
& \cdot\left(\begin{array}{rr}
1 & 1 \\
1 & -1
\end{array}\right), \quad a \neq\{1,-1\}
\end{aligned}
$$

then each block requires only one multiplication plus one at the end to renormalize the result in each channel, that is, a total of $(K+1) / 2$ multiplications per input sample. Note that this is half as much as in the paraunitary case. The number of additions in (66) can actually be reduced by 1 [10]. A hardware structure implementing a linear phase perfect reconstruction filter bank is shown in Fig. 6 . Note that the odd length linear phase filters of Appendix A use $K$ multiplications per input sample, and this for length $2 K+1$ and $2 K+3$ filters. This increased computational load over the even length case is to be expected because of the increased number of free parameters.

Finally, and for completeness, we review the "classical" QMF case. In that case, $H_{1}(z)=H_{0}(-z)$, and $H_{0}(z)$ is a linear phase (symmetric) filter. It can be verified that the corresponding polyphase matrix can be written as

$$
H_{p}(z)=\left(\begin{array}{rr}
1 & 1 \\
1 & -1
\end{array}\right) \prod_{k=1}^{K-1}\left(\begin{array}{ll}
\left(1+\alpha_{k} z^{-1}\right) & \\
& \left(\alpha_{k}+z^{-1}\right)
\end{array}\right) .
$$

Note that perfect reconstruction can only be approximated since the determinant of $H_{p}(z)$ is not a delay (nor a minimum phase filter). Now (67) takes two multiplications

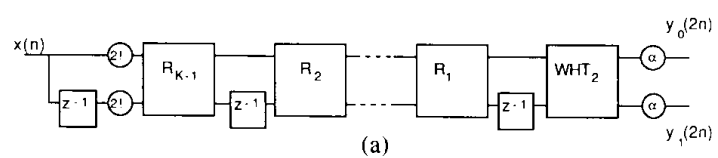

(a)

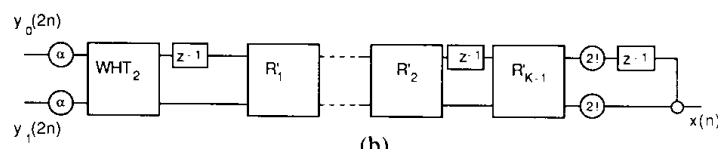

(b)

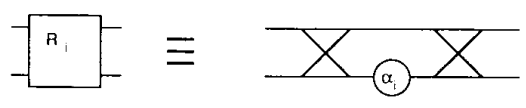

(c)

Fig. 6. (a) Linear phase, perfect reconstruction two-channel analysis filter bank. (b) Equivalent synthesis filter bank. (c) One-multiplier elementary block implementation.

TABLE III

Number of Multiplications for Two-Channel Filter Banks (for Each New InPut and Filters of Length $L=2 K$ )

\begin{tabular}{|c|c|c|}
\hline $\begin{array}{c}\text { Type of } \\
\text { Filter bank }\end{array}$ & $\begin{array}{c}\text { Normalized } \\
\text { block }\end{array}$ & $\begin{array}{c}\text { Denormalized } \\
\text { block }\end{array}$ \\
\hline $\begin{array}{c}\text { min/max phase } \\
\text { (paraunitary) }\end{array}$ & $3 \mathrm{~K} / 2$ & $\mathrm{~K}+1$ \\
Linear Phase & $\mathrm{K}-1$ & $(\mathrm{~K}+1) / 2$ \\
Classical QMF & $\mathrm{K}$ & - \\
\hline
\end{tabular}

per block, that is, assuming a normalization at the output of each channel, a complexity of $K$ multiplications per input sample. This result is well known [47], even without going through a factorization such as (67), but the form of the diagonal matrix in (67) is such that no "denormalization" will reduce the complexity further as it did in the other cases. Table III summarizes the various computational complexities in the two-channel case.

\section{B. Case $N>2$}

Among the sum form solutions derived in Section IV, the pseudo-QMF filter bank with filters of length $L=2 \mathrm{~N}$ [see (27)] is certainly the most efficient in terms of computational complexity. Modulated filter banks have been studied extensively [3], [18] and shown to be equivalent to short filters followed by a fast transform. The complexity of pseudo-QMF filter banks in particular has been studied, for example, in [24]. The short filters correspond to the polyphase filters ( of order $N$ ) of the window function [see (27)], and therefore take on the order of two multiplications per input sample (length- $2 N$ window applied for each set of $N$ new input samples). The fast transform is a type of discrete cosine transform which takes on the order of $N \log (N)$ operations [19], [24], [44], and this for each set of $N$ new input samples. Therefore, the computational complexity for the total pseudo-QMF filter bank is very close to that required by a block transform approach. 
In the product form case (see Section V), the restricted structure of the matrices $\boldsymbol{R}$ can be used to reduce the computational complexity, similarly to what was done in the case $N=2$. For example, the highly constrained case given in (63) can be implemented, in a denormalized version, with only two multiplications.

In conclusion to this section, it was shown that the complexity of the proposed filter banks is in general quite low, and this is because the constraints on the building blocks can be advantageously used in order to reduce the number of arithmetic operations.

\section{Conclusion}

A unified view of some results on perfect reconstruction filter banks has been presented, showing connections and possible cross fertilizations between various approaches. In particular, time-domain and $z$-transform domain approaches have been given and their respective merits were discussed.

Time-domain approaches lead naturally to sum forms of polyphase filter matrices. In that case, a class of windowed filter banks was given, together with a particular solution which is a modulated pseudo-QMF filter bank (similar to the solution given in [25] and [26]). This pseudo-QMF filter bank, while restricted in length ( $L=$ $2 N$ ), achieves both perfect reconstruction and very low computational complexity.

$z$-transform domain approaches lead to product forms for the polyphase filter matrices. In that case, cascaded, paraunitary structures were given that lead to linear phase or pairwise modulated filter banks. Note that such solutions do not exist for the case $N=2$, but become possible for $N>2$. In the important practical case of $N=2$, two structures leading to even or odd length perfect reconstruction linear phase filter banks were proposed.

The computational complexity and structure of the proposed filter banks were shown to be attractive. For example, the linear phase perfect reconstruction filter bank for $N=2$ (even length filters) uses only half as many multiplications as other perfect reconstruction schemes (like the paraunitary filter bank).

As future work, the issue of completeness has to be studied in detail, and the actual design of useful filters has to be undertaken. The generalization of these ideas to the multidimensional case also seems quite promising.

\section{APPENDIX A}

Odd Length Linear Phase Filters for $N=2$

First, note that there are no odd length filters of the same length that will achieve perfect reconstruction [46]. The simplest pair of odd length, linear phase filters is obtained with symmetric filters of length 3 and 5, respectively (the case of length 1 and 3 filters is of no interest). The polyphase filter matrix $\boldsymbol{H}_{p}(z)$ is then given by

$$
\boldsymbol{H}_{p}(z)=\left(\begin{array}{ll}
1+z^{-1} & a \\
1+b z^{-1}+z^{-2} & c\left(1+z^{-1}\right)
\end{array}\right)
$$

The perfect reconstruction constraint requires that Det $\left[\boldsymbol{H}_{p}(z)\right]=z^{-i}$, which leads to $a=c$ as well as to $a$ $\neq 0$ and $b \neq 2$. Thus, with

$$
\begin{aligned}
& H_{0}(z)=1+a z^{-1}+z^{-2} \\
& H_{1}(z)=1+a z^{-1}+b z^{-2}+a z^{-3}+z^{-4}
\end{aligned}
$$

where $a$ and $b$ are different from 0 and 2 , respectively, one has a perfect reconstruction system with a scaling by $a(b-2)$ and a delay of three samples.

More generally, a polyphase matrix $\boldsymbol{H}_{p}(z)$ corresponding to length $2 K+1$ and $2 K+3$ symmetric filters and satisfying perfect FIR reconstruction can be written as

$$
\begin{aligned}
\boldsymbol{H}_{p}(z) & =\left(\begin{array}{ll}
a(z) & b(z) \\
c(z) & d(z)
\end{array}\right) \\
\operatorname{Det}\left[\boldsymbol{H}_{p}(z)\right] & =z^{-K} \\
\text { degree }[a(z)] & =\text { degree }[d(z)]=K \\
\text { degree }[b(z)] & =K-1 \\
\text { degree }[c(z)] & =K+1
\end{aligned}
$$

and the polynomials $a(z), b(z), c(z)$, and $d(z)$ are all "symmetric." A degree- $K$ polynomial $p(z)$ is called "symmetric"' if $z^{-K} p\left(z^{-1}\right)=p(z)$. Note that the product of two symmetric polynomials is symmetric as well as the sum of two symmetric polynomials when they are of the same degree [46].

Now, assume that $\boldsymbol{H}_{p}(z)$ and $\boldsymbol{H}_{p}^{\prime}(z)$ satisfy (A4) with characteristic $K$ and $K^{\prime}$, respectively. Then, it can be verified (using the above properties of symmetric polynomials) that

$$
\boldsymbol{H}_{p}^{\prime \prime}(z)=\boldsymbol{H}_{p}(z) \cdot \boldsymbol{H}_{p}^{\prime}(z)
$$

will satisfy (A4) as well and leads to linear phase filters of length $2\left(K+K^{\prime}\right)+1$ and $2\left(K+K^{\prime}\right)+3$, respectively. Therefore, from the elementary filters in (A2) and (A3), one can write

$$
\begin{gathered}
\boldsymbol{H}_{p}(z)=\prod_{k=1}^{K}\left(\begin{array}{ll}
1+z^{-1} & a_{k} \\
1+b_{k} z^{-1}+z^{-2} & a_{k}\left(1+z^{-1}\right)
\end{array}\right), \\
a_{k} \neq 0, b_{k} \neq 2
\end{gathered}
$$

which leads to length $2 K+1$ and $2 K+3$ linear phase filters. Note that the number of parameters is of the same order as the length of the filters $(2 K)$, that is, twice as many as in the even length case.

By factoring an elementary block as

$$
\left(\begin{array}{ll}
1+z^{-1} & 1 \\
1+b_{k} z^{-1}+z^{-2} & 1+z^{-1}
\end{array}\right) \cdot\left(\begin{array}{ll}
1 & 0 \\
0 & a_{k}
\end{array}\right)
$$

it is seen that the computational complexity is two multiplications per block. Given that there are $K$ blocks and that they are computed at half the input sampling rate, we find a total of $K$ multiplications per input, and this for two channels and filters of length $2 K+1$ and $2 K+3$. 


\section{APPENDIX B}

Generality of the Cascade Structure for Linear Phase Even Length Filters and $N=2$

It is shown that the cascade structure in (44) is only general for filters of length $<8$. For lengths greater or equal to 8 , "singular cases" can be shown which cannot be factored as in (44). In the following, the filters are assumed to be symmetric and antisymmetric with coefficients $a_{i}$ and $b_{i}$, respectively. We assume $a_{0}=b_{0}=1$ without loss of generality (scaling only).

$L=4: H_{p}(z)$ is of the form

$$
\boldsymbol{H}_{p}(z)=\left(\begin{array}{cc}
1+a_{1} z^{-1} & a_{1}+z^{-1} \\
1-b_{1} z^{-1} & b_{1}-z^{-1}
\end{array}\right) .
$$

The determinant equals

$$
\begin{aligned}
\operatorname{Det}\left[\boldsymbol{H}_{p}(z)\right]= & \left(b_{1}-a_{1}\right)+2\left(a_{1} b_{1}-1\right) z^{-1} \\
& +\left(b_{1}-a_{1}\right) z^{-2}
\end{aligned}
$$

from which it follows that $a_{1}=b_{1}$ is the general solution. This is the same as the one generated by (44) (see Table II).

$$
\begin{aligned}
& L=6: \boldsymbol{H}_{p}(z) \text { is of the form } \\
& \boldsymbol{H}_{p}(z)=\left(\begin{array}{cc}
1+a_{2} z^{-1}+a_{1} z^{-1} & a_{1}+a_{2} z^{-1}+z^{-2} \\
1+b_{2} z^{-1}-b_{1} z^{-2} & b_{1}-b_{2} z^{-1}-z^{-2}
\end{array}\right) .
\end{aligned}
$$

So that the determinant is equal to $c z^{-2}$, the following three equations have to be met:

$$
\begin{aligned}
a_{1} & =b_{1} \\
a_{2}\left(a_{1}-1\right) & =b_{2}\left(a_{1}+1\right) \\
a_{2} b_{2}-a_{1} b_{1}+1 & \neq 0 .
\end{aligned}
$$

From Table II, we note that for $L=6$,

$$
\begin{aligned}
& a_{1}=b_{1}=\alpha_{2} \\
& a_{2}=\alpha_{1}\left(\alpha_{2}+1\right) \\
& b_{2}=\alpha_{1}\left(\alpha_{2}-1\right) .
\end{aligned}
$$

Obviously, (A11a) and (A12a) are equivalent. Furthermore, (A11b) means that

$$
b_{2}=a_{2} \frac{\left(a_{1}-1\right)}{\left(a_{1}+1\right)}
$$

which is equivalent to (A12b)-(A12c) with $\alpha_{2}=a_{1}$. Finally, (A11c) becomes, using (A12),

$$
\alpha_{1}^{2}\left(\alpha_{2}^{2}-1\right)-\alpha_{2}^{2}+1=\left(1-\alpha_{1}^{2}\right)\left(1-\alpha_{2}^{2}\right)
$$

that is, equivalent to the scale factor of Table II which has to be different from 0 ( or $\alpha_{1}, \alpha_{2} \neq 1,-1$ ).

$L=8$ : Take, for example, the following two linear phase perfect reconstruction filters:

$$
\begin{aligned}
H_{0}(z)= & 1+z^{-1}+2 z^{-2}+3 z^{-3}+3 z^{-4} \\
& +2 z^{-5}+z^{-6}+z^{-7}
\end{aligned}
$$

$$
\begin{aligned}
H_{1}(z)= & 1+z^{-1}+4 z^{-2}+5 z^{-3}-5 z^{-4} \\
& -4 z^{-5}-z^{-6}-z^{-7} .
\end{aligned}
$$

This is a linear phase perfect reconstruction filter set. However, if one uses (44) in order to synthesize length-8 filters, one notes that $a_{1}=b_{1}=\alpha_{3}$. But here, this would mean that $\alpha_{3}=1$, which leads to a singular block. Therefore, filters like the one in (A15) can only be approximated with the cascade structure in (44). Note that the approximation is numerically ill conditioned (in the example above, the block to be approximated is singular).

\section{ACKNOWLEDGMENT}

This paper benefitted from discussions with a number of people whom the authors would like to thank: H. S. Malvar (University of Brasilia) for discussions on the LOT's, J. Princen (University of Surrey) for his conjecture that pseudo-QMF filters would give perfect reconstruction, S. K. Rao (AT\&T Bell Laboratories) for pointing out some classical network theory results, M. T. J. Smith (Georgia Institute of Technology) for suggesting improvements, and P. P. Vaidyanathan (California Institute of Technology) for his very valuable comments. J. Kovacevic and K. M. Uz (Columbia University) are thanked for their suggestions on the manuscript. The reviewers are thanked for their careful reading of the manuscript and for their constructive criticisms.

\section{REFERENCES}

[1] V. Belevitch, Classical Network Synthesis. San Francisco, CA: Holden-Day, 1968.

[2] R. E. Blahut, Fast Algorithms for Digital Signal Processing. Reading, MA: Addison-Wesley, 1984.

[3] M. G. Bellanger and J. L. Daguet. "TDM-FDM transmultiplexer: Digital polyphase and FFT," IEEE Trans. Commun., vol. COM-22, pp. 1199-1204, Sept. 1974.

[4] P. Cassereau, "A new class of optimal unitary transforms for image processing,"' S.M. thesis, Dep. Elec. Eng. Comput. Sci.. Massachusetts Inst. Technol., Cambridge, May 1985.

[5] P. L. Chu, "Quadrature mirror filter design for an arbitrary number of equal bandwidth channels," IEEE Trans. Acoust., Speech, Signal Processing, vol. ASSP-33, pp. 203-218, Feb. 1985.

[6] R. V. Cox, "The design of uniformly and nonuniformly spaced pseudoquadrature mirror filters," IEEE Trans. Acoust., Speech, Signal Processing, vol. ASSP-34, pp. 1090-1096, Oct. 1986.

[7] R. E. Crochiere and L. R. Rabiner, Multirate Digital Signal Processing. Englewood Cliffs, NJ: Prentice-Hall, 1983.

[8] A. Croisier, D. Esteban, and C. Galand, "Perfect channel splitting by use of interpolation, decimation, tree decomposition techniques," in Proc. Int. Conf. Inform. Sci./Syst., Patras, Greece, Aug. 1976, pp. 443-446.

[9] P. J. Davis, Circulant Matrices. New York: Wiley Interscience, 1979.

[10] Z. Doğanata and P. P. Vaidyanathan, "On one-multiplier implementation of FIR lattice structures." IEEE Trans. Circuits Syst., vol. CAS-34, pp. 1608-1609. Dec. 1987.

[11] C. R. Galand and H. J. Nussbaumer, "Quadrature mirror filters with perfect reconstruction and reduced computational complexity." in Proc. 1985 Int. IEEE Conf. Acoust., Speech, Signal Processing, Tampa, FL, Mar. 1985, pp. 525-529.

[12] T. Kailath, Linear Systems. Englewood Cliffs, NJ: Prentice-Hall, 1980.

[13] D. J. LeGall, "Subband coding of images with low computational complexity," presented at the Picture Coding Symp., Stockholm. Sweden, June 1987

[14] H. S. Malvar, "Optimal pre- and post-filtering in noisy sampled data systems," Ph.D. dissertation, Dep. Elec. Eng. Comput. Sci., Massachusetts Inst. Technol., Cambridge, Aug. 1986. 
[15] J. Masson and Z. Picel, "Flexible design of computationally efficient nearly perfect QMF filter banks," in Proc. 1985 lEEE Conf. Acoust., Speech, Signal Processing, Tampa. FL, Mar. 1985.

[16] F. Mintzer, "Filters for distortion-free two-band multirate filter banks." IEEE Trans. Acoust., Speech, Signal Processing, vol. ASSP33, pp. 626-630, June 1985.

[17] F. D. Murnaghan, The Unitary and Rotation Groups. Washington, DC: Spartan, 1962

[18] M. J. Narasimha and A. M. Peterson, "Design of a 24-channel transmultiplexer," IEEE Trans. Acoust. Speech, Signal Processing, vol. ASSP-27, pp. 752-762, Dec. 1979

[19] _- "On the computation of the discrete cosine transform," IEEE Trans. Commun., vol. COM-26, pp. 934-936, June 1978.

120] K. Nayebi, T. P. Barnwell, and M. J. T. Smith, "Time domain conditions for exact reconstruction in analysis/synthesis systems based on maximally decimated filter banks," in Proc. Southeast. Symp. Syst. Theory, Mar. 1987, pp. 498-503.

[21] T. Q. Nguyen and P. P. Vaidyanathan, "Perfect reconstruction QMF structures which yield linear phase FIR analysis filters," in Proc. Int. Symp. Circuits Syst., Helsinki. Finland, June 1988, pp. 297-300.

[22] H. J. Nussbaumer, "Pseudo-QMF filter bank." IBM Tech. Disclosure Bull., 1981.

[23] - Fast Fourier Transform and Convolution Algorithms. Berlin: Springer, 1982

[24] H. J. Nussbaumer and M. Vetterli, "Computationally efficient QMF filter banks," in Proc. 1984 Int. IEEE Conf. Acoust., Speech, Signal Processing, San Diego, CA, Mar. 1984.

[25] J. Princen and A. Bradley, "Analysis/synthesis filter bank design based on time domain aliasing cancellation," IEEE Trans. Acoust., Speech, Signal Processing, vol. ASSP-34, pp. 1153-1161, Oct. 1986.

[26] J. Princen, A. Johnson, and A. Bradley, "Subband/transform coding using filter bank designs based on time domain aliasing cancellation," in Proc. ICASSP'87, Dallas, TX, Apr. 1987, pp. 2161-2164.

[27] T. A. Ramstad, "Analysisisynthesis filter banks with critical sampling," in Proc. Int. Conf. Digital Signal Processing, Florence, Italy. Sept. 1984, pp. 130-134.

[28] J. H. Rothweiler, "Polyphase quadrature filters-A new subband coding technique," in Proc. 1983 Int. IEEE Conf. Acoust., Speech, Signal Processing, Boston, MA, Mar. 1983, pp. 1280-1283.

[29] M. J. T. Smith and T. P. Barnwell, "A procedure for designing exact reconstruction filter banks for tree structured subband coders," in Proc. IEEE ICASSP' 84 , San Diego, CA, Mar. 1984.

[30] _ . "Exact reconstruction for tree-structured subband coders," IEEE Trans. Acoust., Speech, Signal Processing, vol. ASSP-34, pp. 434441, June 1986.

[31] _ , "A unifying framework for analysis/synthesis systems based on maximally decimated filter banks," in Proc. ICASSP'85, Tampa, FL. Mar. 1985 , pp. $52 \mathrm{I}-524$.

[32] _ . "A new filter bank theory for time-frequency representation," IEEE Trans. Acoust., Speech, Signal Processing, vol. ASSP-35, pp. 314-327, Mar. 1987

[33] P. P. Vaidyanathan and S. K. Mitra, "A general family of multivariable digital lattice filters," IEEE Trans. Circuits Syst, vol. CAS32, pp. 1234-1245, Dec. 1985.

[34] P. P. Vaidyanathan, "Theory and design of $M$-channel maximally decimated quadrature mirror filters with arbitrary $M$, having perfect reconstruction property," IEEE Trans. Acoust., Speech. Signal Processing, vol. ASSP-35, pp. 476-492, Apr. 1987

[35] P. P. Vaidyanathan and P.-Q. Hoang, "The perfect reconstruction QMF bank: New architectures, solutions, and optimization strategies," in Proc. IEEE Int. Conf. Acoust., Speech, Signal Processing, Dallas, TX, Apr. 1987, pp. 2169-2172.

136] P. P. Vaidyanathan. Z. Doganata, and T. Q. Nguyen, "More results on the perfect reconstruction problem in $M$-band parallel QMF banks," in Proc. IEEE Int. Symp. Circuits Syst., Philadelphia, PA, May 1987, pp. 847-850.

[37] P. P. Vaidyanathan. "Quadrature mirror filter banks, $M$-band extensions and perfect-reconstruction technique," IEEE ASSP Mag., vol. 4. pp. 4-20, July 1987.
[38] P. P. Vaidyanathan, Z. Doğanata, and T. Q. Nguyen, "General synthesis procedures for FIR lossless transfer matrices, for perfect-re construction multirate filter bank applications," Dep. Elec. Eng., California Inst. Technol., Pasadena, Tech. Rep., Aug. 1987. See also IEEE Trans. Acoust., Speech, Signal Processing, vol. 36, pp. 15611574, Oct. 1988.

[39] P. P. Vaidyanathan and P.-Q. Hoang, "Lattice structures for optimal design and robust implementation of two-band perfect reconstruction QMF banks," IEEE Trans. Acoust., Speech, Signal Processing, vol. 36, pp. 81-94, Jan. 1988.

[40] P. P. Vaidyanathan and S. K. Mitra, "Polyphase networks, block digital filtering, LPTV systems, and alias-free QMF banks: A unified approach based on pseudocirculants," IEEE Trans. Acoust., Speech, Signal Processing, vol. 36, pp. 381-391, Mar. 1988.

[41] P. P. Vaidyanathan and Z. Doğanata, "The role of lossless systems in modern digital signal processing: A tutorial," IEEE Trans. Educ., to appear, Aug. 1989

[42] P. P. Vaidyanathan, private communication, June 1988

[43] M. Vetterli, "Multidimensional subband coding: Some theory and algorithms," Signal Processing, vol. 6, pp. 97-112, Feb. 1984

[44] M. Vetterli and H. J. Nussbaumer, "Simple FFT and DCT algorithms with reduced number of operations,' Signal Processing, vol. 6, pp. 267-278, Aug. 1984.

[45] $M$. Vetterli, "Splitting a signal into subsampled channels allowing perfect reconstruction," in Proc. IASTED Conf. Appl. Signal Processing Digital Filtering, Paris, France, June 1985.

[46] — , "Filter banks allowing perfect reconstruction," Signal Processing, vol. 10, pp. 219-244, Apr. 1986.

[47] — "A theory of multirate filter banks," IEEE Trans. Acoust. Speech, Signal Processing, vol. ASSP-35, pp. 356-372. Mar. 1987

[48] M. Vetterli and D. Le Gall, "Perfect reconstruction FIR filter banks: Lapped transforms, pseudo-QMF's and paraunitary matrices," in Proc. Int. Symp. Circuits Syst., Helsinki, Finland, June 1988, pp. 2249-2253.

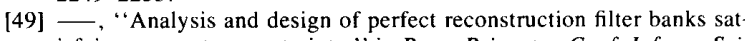
isfying symmetry constraints," in Proc. Princeton Conf. Inform. Sci. Syst., Mar. 1988, pp. 670-675.

[50] E. Viscito and J. Allebach, "The design of tree-structured $M$-channe filter banks using perfect reconstruction filter blocks," in Proc. ICASSP'88, New York, NY, Apr. 1988, pp. 1475-1478.

Martin Vetterli (S'86-M'86), for a photograph and biography, see p. 64 of the January 1989 issue of this TRANSACTIONS

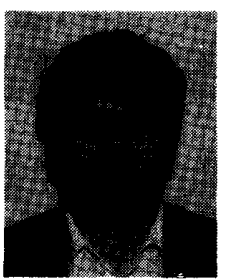

Didier Le Gall (M'88) was born in Paris, France, in 1954. He received the Diplome d'Ingenieur from Ecole Centrale de Lyon, Ecully, France, in 1976, and the M.S. and Ph.D. degrees in electrical engineering from the University of California at Los Angeles (UCLA) in 1977 and 1981, respectively.

From 1982 to 1985 he was with the Medical Imaging Division of Thomson CSF, Paris, France, where he pursued research in a reconstruction and display algorithm for computerized tomography. In 1985 he joined Bell Communications Research, Morristown, NJ, firs as a member of the Technical Staff working on signal processing applied to image communications, then as District Manager of the Visual Communications Group. His research interests lie in the field of signal processing, filter banks, digital image and video compression, as well as high definition television. He is also an Adjunct Professor in the Department of Electrical Engineering at Columbia Univeristy, New York. 